\title{
MONOTONICITY AND SEPARATION FOR THE MUMFORD-SHAH PROBLEM
}

\author{
MONOTONIE ET SÉPARATION DANS \\ LE PROBLÈME DE MUMFORD-SHAH
}

\begin{abstract}
Guy DAVID, Jean-Christophe LÉGER
Mathématiques, Bâtiment 425, Université de Paris-Sud, 91405 Orsay Cedex, France
\end{abstract}

Received 9 April 2001

ABSTRACT. - We prove some monotonicity properties of the global Mumford-Shah minimizers as defined by Bonnet in [4]. The main consequence is that the only solutions for which the complement in the plane of the singular set is not connected correspond to lines and propellers. We also get a boundary version of the Mumford-Shah conjecture.

(C) 2002 L'Association Publications de l'Institut Henri Poincaré. Published by Elsevier B.V. All rights reserved

RÉSUMÉ. - Nous prouvons des propriétés de monotonie des solutions du problème de Mumford-Shah global défini par Bonnet dans [4]. La conséquence principale en est que les seules solutions dont le complémentaire de l'ensemble singulier n'est pas connexe correspondent à des droites et des propulseurs. Nous obtenons aussi une version à la frontière de la conjecture de Mumford-Shah.

(C) 2002 L'Association Publications de l'Institut Henri Poincaré. Published by Elsevier B.V. All rights reserved AMS classification: 49K99; 49Q20

\section{Introduction}

The main concern in this paper is the study of minimizers for the global MumfordShah problem in the plane. The precise notion, introduced by A. Bonnet in [4], is as follows.

Let $K \subset \mathbb{R}^{2}$ be a closed subset of the plane, and $u \in W_{\text {loc }}^{1,2}\left(\mathbb{R}^{2} \backslash K\right)$ a real-valued function defined on the open set $\mathbb{R}^{2} \backslash K$ and whose distributional derivative there lies

E-mail addresses: guy.david@math.u-psud.fr (G. David), jean-christophe.leger@math.u-psud.fr (J.-C. Léger). 
in $L_{\mathrm{loc}}^{2}\left(\mathbb{R}^{2} \backslash K\right)$. We say that $(u, K)$ is an admissible pair if in addition

$$
H^{1}(K \cap B(0, R))+\int_{B(0, R) \backslash K}|\nabla u|^{2}<+\infty
$$

for all $R>0$. Here $H^{1}$ denotes the 1-dimensional Hausdorff measure (which is the appropriate generalization of arclength measure); see for instance [12,13,18]. Also, our convention in this paper is that $B(0, R)$ denotes the open disk with center 0 and radius $R$.

Let $(u, K)$ be an admissible pair. A competitor for $(u, K)$ is an admissible pair $(v, L)$ such that, for $R$ large enough,

$$
\begin{gathered}
L \backslash B(0, R)=K \backslash B(0, R), \\
v(x)=u(x) \quad \text { for } x \in \mathbb{R}^{2} \backslash(K \cup B(0, R)),
\end{gathered}
$$

and

$$
\left\{\begin{array}{l}
\text { if } x, y \in \mathbb{R}^{2} \backslash(K \cup B(0, R)) \text { lie in different connected components of } \\
\mathbb{R}^{2} \backslash K, \text { then they lie in different connected components of } \mathbb{R}^{2} \backslash L \text { as well. }
\end{array}\right.
$$

Thus $(v, L)$ coincides with $(u, K)$ out of some bounded set, and also $L$ separates points near infinity at least as well as $K$ does.

DEFINITION 1.5. - A global Mumford-Shah minimizer in the plane is an admissible pair $(u, K)$ such that if $(v, L)$ is any competitor for $(u, K)$, then

$$
H^{1}(K \cap B(0, R))+\int_{B(0, R) \backslash K}|\nabla u|^{2} \leqslant H^{1}(L \cap B(0, R))+\int_{B(0, R) \backslash L}|\nabla v|^{2}
$$

for $R$ large enough.

There is a minor issue that needs to be addressed. If $(u, K)$ is an admissible pair, we say that it is reduced if there is no competitor $(v, L)$ for $(u, K)$ such that $L \subset K, L \neq K$, and $v(x)=u(x)$ for $x \in \mathbb{R}^{2} \backslash K$. It is not hard to check that for each admissible pair $(u, K)$ there is a reduced admissible pair $(v, L)$ such that $L \subset K, v$ is an extension of $u$ (as above), and (1.4) holds for all $R$. Because of this, we shall restrict our attention to reduced global minimizers, without loss of generality. The point of this reduction is that we may now have cleaner statements on $K$ (otherwise, we could make it ugly artificially by adding a set of vanishing Hausdorff measure to it).

Notation 1.7. - To save some space we shall denote by RGM the set of reduced global Mumford-Shah minimizers in the plane.

The main reason for introducing RGM is that limits under blow-up procedures of (usual) reduced Mumford-Shah minimizers in planar domains lie in RGM. Incidentally, this is the reason for the topological constraint (1.4) on competitors, which comes 
indirectly from normalizing out additive constants in the blow-up procedure. See [4] for details about this.

Of course understanding RGM well would help in the study of usual Mumford-Shah minimizers. This is not the main topic of this paper, but we shall rapidly discuss an instance of this in Section 13.

Here is a short list of known pairs $(u, K) \in R G M$ :

$$
K=0 \text { and } u \text { is constant on } \mathbb{R}^{2} \text {; }
$$

$K$ is a line and $u$ is constant on each connected component of $\mathbb{R}^{2} \backslash K$;

$K$ is a propeller (i.e., the union of 3 closed half-lines with a common extremity and that make $120^{\circ}$ angles at that point) and $u$ is a constant on each of the 3 components of $\mathbb{R}^{2} \backslash K$;

there is a set of Euclidean coordinates in $\mathbb{R}^{2}$ where $K=\mathbb{R}^{+}=\{(x, 0) ; x \geqslant 0\}$ and $u(r \cos \theta, r \sin \theta)=C \pm \sqrt{2 / \pi} r^{1 / 2} \cos (\theta / 2)$ for $r>0$ and $0<\theta<2 \pi$.

Of course the values of the constant $C$ and the constant sign \pm in (1.11) do not matter. The fact that the pairs in (1.8)-(1.10) are global minimizers is rather easy to check directly. For (1.11), this is the main result in [5].

It is reasonable to conjecture that the short list (1.8)-(1.11) is complete, i.e., that there is no other reduced global minimizer. This would imply the Mumford-Shah conjecture from [19] that says that for reduced minimizers of the Mumford-Shah functional on simple planar domains (see the definition at the begining of Section 13), the singular set $K$ is a finite union of $C^{1}$ curves. The argument for the implication is similar to the one in [4] for isolated connected components of $K$, but does not seem to be written explicitely anywhere yet.

Let us rapidly remind the reader of some of the known facts about RGM. From now on, we shall always assume that $(u, K) \in R G M$.

First, it is easy to see that $u$ is harmonic on $\mathbb{R}^{2} \backslash K$. It also satisfies the Neumann boundary condition $\frac{\partial u}{\partial n}=0$ on $K$ (which happens to make sense). This is just because $u$ minimizes the energy $\int_{\mathbb{R}^{2} \backslash K}|\nabla u|^{2}$ locally.

Also, $u$ is essentially uniquely determined by $K$ (that is, modulo adding a constant to $u$ and multiplying it by \pm 1 in each component of $\mathbb{R}^{2} \backslash K$ ). There is even a formula that allows us to compute the square of the complex derivative $\left(\frac{\partial u}{\partial z}\right)^{2}$ in terms of $K$. See [15].

Next, if $K$ is not empty, it is Ahlfors-regular and uniformly rectifiable. This comes essentially from [6] and [9]. We even know from a minor modification of [7] or [3] that

for $H^{1}$-almost every point $x \in K$, there is a disk $B(x, r)$ such that

$$
K \cap B(x, r) \text { is a } C^{1} \text { curve though } B(x, r) .
$$

We shall call such a point $x$ a "regular point" of $K$. 
The words "essentially" and "a minor modification of" in the previous two sentences come from the fact that in $[6,9,7,3]$, the topological condition (1.4) was not considered. But this is not a serious issue.

In the special case when $K$ is connected, it was proved by A. Bonnet that $(u, K)$ is one of the pairs described in (1.8)-(1.11). See [4].

One of the main goals of this paper is to show that if $\mathbb{R}^{2} \backslash K$ is not connected, then $(u, K)$ is as in our examples (1.9) or (1.10). Thus we shall be left with the problem of finding the global minimizers $(u, K)$ for which $K$ is not connected (because of Bonnet) and $\mathbb{R}^{2} \backslash K$ is connected.

Note that when $\mathbb{R}^{2} \backslash K$ is connected, the topological condition (1.4) is automatically satisfied; thus we shall be left with the same minimizing problem as mentioned by De Giorgi [10].

Our proof will be based on a monotonicity argument a little like the one in [4], but we shall use a mixture $2 E+\ell$ of energy and length, instead of the energy $E$ alone.

We shall also obtain some information when $\mathbb{R}^{2} \backslash K$ is connected. For instance, the case when $K$ has a central symmetry is easy to deal with. See Section 9 for this and a few similar results.

Our argument will give new information on $K_{0}$ when $K_{0}$ is a connected component of $\mathrm{K}$ which is not reduced to one point. We shall see in Section 10 that $K_{0}$ is a "chordarc tree" composed of $C^{1}$ curves that can only meet by sets of 3 and with $120^{\circ}$ angles. But we do not know at this point if there can be infinitely many such curves; they may possibly accumulate at the ends of $K_{0}$, as suggested by Fig. 1 . A more precise description of $K_{0}$, and also estimates on the size of the jump of $u$ at points of $K_{0}$, will be given in Section 10.

As a simple application, we shall see in Section 11 that the set $K^{\sharp} \subset K$ of points of high energy is stable when we take limits of reduced global minimizers.

Consider the conic sector $C_{\alpha}=\left\{(\rho \cos \theta, \rho \sin \theta) \in \mathbb{R}^{2} ; \rho>0\right.$ and $\left.0<\theta<\alpha\right\}$ in the plane. We can define global minimizers in $C_{\alpha}$ as we did in the plane, and it turns out that when $0<\alpha<3 \pi / 2$ we can give a simple description of all (reduced) global minimizers in $C_{\alpha}$. See Section 12.

This include the case of half-planes (when $\alpha=\pi$ ), which we can use to give a good description of the boundary behaviour of the (usual) reduced Mumford-Shah minimizers in bounded smooth domains in the plane. See Section 13 for a rapid description of how it works. A similar result has been shown independently and simultaneously by Maddalena and Solimini in [17].

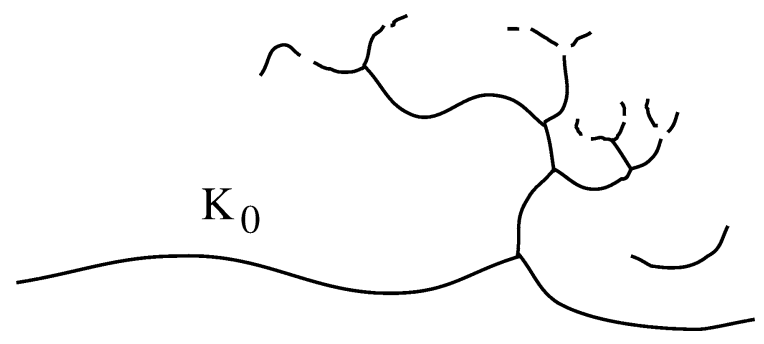

Fig. 1. 


\section{Notations and the monotonicity statements}

Let a reduced global minimizer $(u, K) \in R G M$ be given. We consider the functions

$$
E(r)=\int_{B(0, r) \backslash K}|\nabla u|^{2}
$$

and

$$
\ell(r)=H^{1}(K \cap B(0, r)),
$$

where we recall that $B(0, r)$ is the open disk centered at 0 and with radius $r$. Since everything is invariant under translations, the choice of origin does not matter. The main point of the argument that will follow is to show that in many circumstances,

$$
F(r)=\frac{\ell(r)+2 E(r)}{r}
$$

is an increasing function of $r$. Note that $F(r)$ is normalized to be scale-invariant. It is also bounded, because

$$
E(r)+\ell(r) \leqslant 2 \pi r \quad \text { for } r>0 .
$$

This is a very easy and classical estimate obtained by comparing $(u, K)$ with the competitor $(v, L)$, where $L=K \cup \partial B(0, r) \backslash B(0, r), v(x)=u(x)$ out of $B(0, r) \cup L$, and $v(x)=0$ on $B(0, r)$.

Our first proposition says that there is an open set $\mathcal{R}$ of full Lebesgue measure where $F$ is differentiable, and $F$ increases at least as fast as its derivative on $\mathcal{R}$ suggests.

Let $\mathcal{R}$ denote the set of $r>0$ such that there is a neighborhood of $\partial B(0, r)$ in which $K$ is composed of finitely many $C^{1}$ curves that all meet $\partial B(0, r)$ transversally only. (Thus $K \cap \partial B(0, r)$ is finite when $r \in \mathcal{R}$.)

Proposition 2.5. - The set $\mathcal{R}$ is open, $H^{1}((0,+\infty) \backslash \mathcal{R})=0, F$ is continuously differentiable on $\mathcal{R}$, and

$$
F(b)-F(a) \geqslant \int_{\mathcal{R} \cap(a, b)} F^{\prime}(r) d r \quad \text { for } 0<a<b<+\infty .
$$

This will be proved later in this section, and at the same time $F^{\prime}(r)$ will be computed. For the moment we want to give a few statements with lower bounds on $F^{\prime}(r), r \in \mathcal{R}$, which will be proved in later sections. We shall distinguish between cases, depending on the number $N(r)$ of points in $K \cap \partial B(0, r)$.

PROPOSITION 2.7. - If $K \cap \partial B(0, r)$ is empty,

$$
r F^{\prime}(r) \geqslant \int_{\partial B(0, r)}|\nabla u|^{2} .
$$

Unfortunately we shall not prove any good monotonicity estimate when $N(r)=1$. 
PROPOSITION 2.9. - Let $r \in \mathcal{R}$ be such that $N(r) \geqslant 2$. If $N(r)=2$, suppose in addition that

the two points of $K \cap \partial B(0, r)$ lie in the same connected component of $K$.

Then

$$
r F^{\prime}(r) \geqslant 10^{-10} \int_{\partial B(0, r) \backslash K}|\nabla u|^{2} .
$$

Proposition 2.12. - If $r \in \mathcal{R}$ and $N(r) \geqslant 4$, then $r F^{\prime}(r) \geqslant 10^{-3}$.

PROPOSITION 2.13. - Let $r \in \mathcal{R}$ be such that all the connected components of $\partial B(0, r) \backslash K$ have lengths at most $\pi \omega r$ for some $\omega \leqslant 3 / 2$. Then

$$
r F^{\prime}(r) \geqslant \min \{1,3-2 \omega\} \int_{\partial B(0, r) \backslash K}|\nabla u|^{2} .
$$

All these estimates will be proved in the next three sections, but let us already check Proposition 2.5. and compute $r F^{\prime}(r)$ here.

It is clear from its definition that $\mathcal{R}$ is open. Denote by $K^{*}$ the set of regular points of $K$, i.e., points that satisfy the property in (1.12). Thus $H^{1}\left(K \backslash K^{*}\right)=0$, by (1.12). Then

$$
\partial B(0, r) \cap\left(K \backslash K^{*}\right)=\emptyset
$$

for $H^{1}$-almost every $r>0$, because the set where this fails is the image of $K \backslash K^{*}$ under the Lipschitz mapping $x \rightarrow|x|$. Next, the set of radii $r$ such that

$$
\partial B(0, r) \text { meets } K^{*} \text { tangentially at least once }
$$

is composed of critical values of the function $|x|$ on a countable union of $C^{1}$ curves, hence it has vanishing $H^{1}$-measure by Sard's theorem.

When $r$ satisfies (2.15) but not (2.16), $K$ can only meet $\partial B(0, r)$ on $K^{*}$ and nontangentially. In particular, $K \cap \partial B(0, r)$ is finite; then it is easy to see that $r \in \mathcal{R}$. This proves that $H^{1}((0,+\infty) \backslash \mathcal{R})=0$.

Next we want to study $F^{\prime}$. Let us start with the function $E$. If we write the integral in (2.1) in polar coordinates and then use Fubini, we see that

$$
E^{\prime}(r)=\int_{\partial B(0, r) \backslash K}|\nabla u|^{2} d H^{1}
$$

not only almost-everywhere, but also in the sense of distributions. That is, $E(r)$ in the indefinite integral of its almost-everywhere derivative $E^{\prime}(r)$. Then we easily get that

$$
\left(\frac{E(r)}{r}\right)^{\prime}=\frac{1}{r} \int_{\partial B(0, r) \backslash K}|\nabla u|^{2}-\frac{E(r)}{r^{2}}
$$


almost-everywhere and in the sense of distributions.

This will be enough for our purposes, but since we announced that $F$ is continuously differentiable on $\mathcal{R}$, let us rapidly say why this is the case for $E(r)$ (and hence $\frac{E(r)}{r}$ ). The point is that $u$ is harmonic on $\mathbb{R}^{2} \backslash K$, but also $\nabla u$ has continuous extensions up to the boundary on both sides of $K$ near a regular point of $K$. This is not worth insisting too much, though.

Next, we want to compute $\ell^{\prime}(r)$. Note that $\ell(r)$ is a nondecreasing function of $r$, so it is differentiable almost-everywhere and its distributional derivative is the sum of $\ell^{\prime}(r)$ (the absolutely continuous part), plus perhaps a positive singular measure that we do not need to know precisely. Then the distributional derivative of $\frac{\ell(r)}{r}$ on $(0,+\infty)$ is $\frac{\ell^{\prime}(r)}{r}-\frac{\ell(r)}{r^{2}}$, plus perhaps a positive measure. In particular,

$$
\frac{\ell(b)}{b}-\frac{\ell(a)}{a} \geqslant \int_{a}^{b}\left(\frac{\ell^{\prime}(r)}{r}-\frac{\ell(r)}{r^{2}}\right) d r \quad \text { for } 0<a<b<+\infty .
$$

The derivative $\ell^{\prime}(r)$ is easy to compute when $r \in \mathcal{R}$. For $r \in \mathcal{R}$ and $x \in K \cap \partial B(0, r)$, call $\alpha(x) \in[0, \pi / 2)$ the (non oriented) angle between the radius $[0, x]$ and the tangent line to $K$ at $x$. Then

$$
\ell^{\prime}(r)=\sum_{x \in K \cap \partial B(0, r)} \frac{1}{\cos \alpha(x)} \quad \text { for } r \in \mathcal{R} .
$$

Thus $\frac{\ell(r)}{r}$ is continuously differentiable on $\mathcal{R}$. To complete our proof of Proposition 2.5 we just need to check (2.6), which follows directly from (2.19) and its analogue for $\frac{E(r)}{r}$. Our computation also says that for $r \in \mathcal{R}$,

$$
\begin{aligned}
F^{\prime}(r) & =\frac{2}{r} \int_{\partial B(0, r) \backslash K}|\nabla u|^{2}-\frac{2 E(r)}{r^{2}}+\frac{\ell^{\prime}(r)}{r}-\frac{\ell(r)}{r^{2}} \\
& =\frac{2}{r} \int_{\partial B(0, r) \backslash K}|\nabla u|^{2}+\frac{1}{r} \sum_{x \in K \cap \partial B(0, r)} \frac{1}{\cos \alpha(x)}-\frac{2 E(r)+\ell(r)}{r^{2}},
\end{aligned}
$$

by (2.18) and (2.20).

In the next section we derive simpler expressions for $F^{\prime}(r), r \in \mathcal{R}$, which will then be used in later sections to prove Propositions 2.7-2.13.

\section{How to go from normal to tangential derivatives}

The integral in (2.21) contains normal and tangential derivatives. We shall see later that tangential derivatives are much easier to use than normal ones when we want estimates on $E(r)+\ell(r)$. In this section we prove a formula that will allow us to trade normal derivatives for tangential ones. 
We need more notation. Let us use polar coordinates $(r, \theta)$ and write

$$
|\nabla u|^{2}=\left(\frac{\partial u}{\partial \tau}\right)^{2}+\left(\frac{\partial u}{\partial v}\right)^{2}
$$

where $\frac{\partial u}{\partial \tau}=\frac{1}{r} \frac{\partial u}{\partial \theta}$ is the tangential derivative and $\frac{\partial u}{\partial v}=\frac{\partial u}{\partial r}$ is the radial derivative of $u$. Then

$$
\int_{\partial B(0, r) \backslash K}|\nabla u|^{2}=J_{\tau}+J_{v},
$$

where we set

$$
\begin{aligned}
& J_{\tau}=\int_{\partial B(0, r) \backslash K}\left(\frac{\partial u}{\partial \tau}\right)^{2} d H^{1}, \\
& J_{v}=\int_{\partial B(0, r) \backslash K}\left(\frac{\partial u}{\partial v}\right)^{2} d H^{1} .
\end{aligned}
$$

Proposition 3.5. - We have that

$$
J_{v}=J_{\tau}-\frac{\ell(r)}{r}+\sum_{x \in \partial B(0, r) \cap K} \cos \alpha(x)
$$

for $r \in \mathcal{R}$, where $\ell(r), \mathcal{R}$, and $\alpha(x)$ are as in (2.2), Proposition 2.5, and near (2.20).

Before we prove the proposition let us see how to use it to compute $F^{\prime}(r)$. From $(2.21)$ we deduce that

$$
\begin{aligned}
r F^{\prime}(r) & =2 J_{\tau}+2 J_{v}+\sum_{x \in K \cap \partial B(0, r)} \frac{1}{\cos \alpha(x)}-\frac{2 E(r)+\ell(r)}{r} \\
& =3 J_{\tau}+J_{v}+\sum_{x \in K \cap \partial B(0, r)}\left\{\frac{1}{\cos \alpha(x)}+\cos \alpha(x)\right\}-2\left(\frac{E(r)+\ell(r)}{r}\right)
\end{aligned}
$$

Recall that $N(r)$ is the number of points in $K \cap \partial B(0, r)$. Since $\frac{1}{\cos \alpha(x)}+\cos \alpha(x) \geqslant 2$ for all $x$, we get that

$$
\frac{r}{2} F^{\prime}(r) \geqslant \frac{3}{2} J_{\tau}+\frac{1}{2} J_{v}+N(r)-\frac{E(r)+\ell(r)}{r} .
$$

This is the estimate that will be used most of the time, but in some cases we may choose to replace one $J_{\tau}$ with $J_{v}$ in the first line of (3.7) and get that

$$
\begin{aligned}
r F^{\prime}(r) & =J_{\tau}+3 J_{v}+\sum_{x \in K \cap \partial B(0, r)}\left\{\frac{1}{\cos \alpha(x)}-\cos \alpha(x)\right\}-\frac{2 E(r)}{r} \\
& \geqslant J_{\tau}+3 J_{v}-\frac{2 E(r)}{r} .
\end{aligned}
$$


Now we start to prove Proposition 3.5. Our original proof was a variant of an argument from [15]. The idea was to identify $\mathbb{R}^{2}$ with $\mathbb{C}$ and use the formula

$$
\left(\frac{\partial u}{\partial x}-\mathrm{i} \frac{\partial u}{\partial y}\right)^{2}(w)=-\frac{1}{2 \pi} \int_{K} \frac{d H^{1}(z)}{(z-w)^{2}} \quad \text { for } w \in \mathbb{C} \backslash K
$$

on the complex derivative of $u$.

The proposition can be derived by computing $\int_{\partial B(0, r) \backslash K}\left(\frac{\partial u}{\partial z}(w)\right)^{2} w^{2} d H^{1}(w)$ in two different ways, and in particular using (3.10) and residues.

The proof that we shall give here is a little longer, but more direct. It has the advantage of working as soon as $(u, K)$ is a local minimizer in some neighborhood of $\bar{B}(0, r)$. That is, we shall only need to know that (1.6) holds for competitors $(v, L)$ that coincide with $(u, K)$ out of some (fixed) neighborhood of $\bar{B}(0, r)$. We shall also use in Section 12 the fact that the proof still works when the plane is replaced with some cone $C_{\alpha}=\{(\rho \cos \theta, \rho \sin \theta) ; \rho>0$ and $0<\theta<\alpha\}$ for some $\alpha \leqslant 2 \pi$ (that is, when $K \subset C_{\alpha}$ and $u$ is defined on $C_{\alpha} \backslash K$ ).

We wish to thank F. Maddalena and S. Solimini, who found out about Proposition 3.5 independently, for telling us that there is a direct variational proof.

Note that it is enough to prove (3.6) when $r=1$. This follows from a standard homogeneity argument, that we shall use a few times in this paper. The point is that if we set $K_{\lambda}=\lambda K$ and $u_{\lambda}(x)=\lambda^{1 / 2} u\left(\frac{x}{\lambda}\right)$ for $x \in \mathbb{R}^{2} \backslash K_{\lambda}$, then $\left(u_{\lambda}, K_{\lambda}\right) \in R G M$ for all choices of $\lambda>0$. It is easy to see that both sides of (3.6) are preserved when we replace $(u, K)$ with $\left(u_{\lambda}, K_{\lambda}\right)$ (and modify $r$ accordingly).

So we take $r=1$, which we assume to lie in $\mathcal{R}$. Later in the proof the letter $r$ will appear again, but it will denote other variables; hopefully this will not create any confusion.

We want to construct a one-parameter family of competitors $\left(u_{t}, K_{t}\right)$ for $(u, K)$, but first we want to construct homeomorphisms $\varphi_{t}$. Let $r_{0} \in(0,1)$ be a parameter, which we intend to send to 1 at the end of the argument. Since $1 \in \mathcal{R}$, we know that for $r_{0}$ close enough to 1 (which we shall assume), the intersection of $K$ with $A=B(0,1) \backslash B\left(0, r_{0}\right)$ is composed of finitely many $C^{1}$ arcs, none of which is ever tangent to a circle $\partial B(0, r)$, $r_{0} \leqslant r \leqslant 1$. First set

$$
f(r)= \begin{cases}r & \text { for } 0 \leqslant r \leqslant r_{0}, \\ \frac{r_{0}(1-r)}{1-r_{0}} & \text { for } r_{0} \leqslant r \leqslant 1, \\ 0 & \text { for } r \geqslant 1 .\end{cases}
$$

Note that $f$ is continuous on $[0,+\infty)$. Next define $g=g_{t}$ for $t \in \mathbb{R}$ by

$$
g_{t}(r)=r+t f(r) \quad \text { for } r \geqslant 0 .
$$

We shall only be interested in small values of $t$, and for these $g_{t}$ is a piecewise affine bijection of $[0,+\infty)$, with

$$
\frac{1}{2} \leqslant g_{t}^{\prime}(r) \leqslant 2 \text { and } \quad \frac{r}{2} \leqslant g_{t}(r) \leqslant 2 r .
$$


Now define a piecewise $C^{1}$ diffeomorphism $\varphi=\varphi_{t}$ of $\mathbb{R}^{2}$ by

$$
\varphi_{t}(r \cos \theta, r \sin \theta)=\left(g_{t}(r) \cos \theta, g_{t}(r) \sin \theta\right) .
$$

Finally set

$$
K_{t}=\varphi_{t}(K) \quad \text { and } \quad u_{t}(x)=u\left(\varphi_{t}^{-1}(x)\right) \text { for } x \in \mathbb{R}^{2} \backslash K_{t} .
$$

It is easy to see that for $t$ small enough $\left(u_{t}, K_{t}\right)$ is a competitor for $(u, K)$. In particular, $\left(u_{t}, K_{t}\right)$ coincides with $(u, K)$ out of $B(0,1)$ because $f(r)=0$ for $r \geqslant 1$. Set

$$
a(t)=H^{1}\left(K_{t} \cap B(0,1)\right)
$$

and

$$
e(t)=\int_{B(0,1) \backslash K_{t}}\left|\nabla u_{t}\right|^{2} .
$$

Then

$$
a(0)+e(0) \leqslant a(t)+e(t)
$$

for $t$ small, because $\left(u_{0}, K_{0}\right)=(u, K)$ and $(u, K)$ is a global minimizer (see Definition 1.5).

Next we want to check that $a(t)$ and $e(t)$ have derivatives at $t=0$, and compute $a^{\prime}(0)$ and $e^{\prime}(0)$. Set $B=B\left(0, r_{0}\right)$. Note that

$$
\varphi_{t}(x)=(1+t) x \quad \text { for } x \in B \text {, by (3.11), (3.12), and (3.14). }
$$

Then

$$
H^{1}\left(\varphi_{t}(K \cap B)\right)=(1+t) H^{1}(K \cap B) .
$$

Next consider $K \cap A=K \cap[B(0,1) \backslash B]$, and use polar coordinates. Notice that $\varphi$ preserves the radial and tangential directions; the size of its derivatives in these directions is

$$
\frac{\partial \varphi_{\mathrm{rad}}}{\partial v}=g^{\prime}(r) \quad \text { and } \quad \frac{\partial \varphi_{\mathrm{tan}}}{\partial \tau}=\frac{g(r)}{r} .
$$

Let us still denote by $\alpha(x) \in[0, \pi / 2]$ the angle between $[0, x]$ and the tangent line to $K$ at $x$. It is well defined for $x \in K \cap A$, because we restricted to $r_{0}$ close to 1 . Then

$$
H^{1}\left(\varphi_{t}(K \cap A)\right)=\int_{K \cap A} h_{t}(x) d H^{1}(x),
$$

with

$$
h_{t}(x)=\left\{g_{t}^{\prime}(r)^{2} \cos ^{2} \alpha(x)+\left(\frac{g_{t}(r)}{r}\right)^{2} \sin ^{2} \alpha(x)\right\}^{1 / 2} .
$$

Note that $g_{t}^{\prime}(r)$ and $g_{t}(r) / r$ stay reasonably close to 1 , by (3.13). Thus we can differentiate (3.22) under the integral sign. Here 


$$
\frac{\partial}{\partial t}\left(h_{t}(x)\right)=h_{t}(x)^{-1}\left\{\frac{\partial}{\partial t}\left(g_{t}^{\prime}(r)\right) g_{t}^{\prime}(r) \cos ^{2} \alpha(x)+\frac{\partial}{\partial t}\left(\frac{g_{t}(r)}{r}\right) \frac{g_{t}(r)}{r} \sin ^{2} \alpha(x)\right\}
$$

and since

$$
g_{t}^{\prime}(r)=1+t f^{\prime}(r), \quad \frac{g_{t}(r)}{r}=1+t \frac{f(r)}{r}
$$

by $(3.12)$, we get that

$$
\frac{\partial}{\partial t}\left(h_{t}(x)\right)_{\mid t=0}=f^{\prime}(r) \cos ^{2} \alpha(x)+\frac{f(r)}{r} \sin ^{2} \alpha(x),
$$

because $g_{t}(x) \equiv x$ for $t=0$. Finally

$$
\begin{aligned}
a^{\prime}(0) & =H^{1}(K \cap B)+\left.\frac{\partial}{\partial t}\left(H^{1}\left(\varphi_{t}(K \cap A)\right)\right)\right|_{t=0} \\
& =H^{1}(K \cap B)+\left.\int_{K \cap A} \frac{\partial}{\partial t}\left(h_{t}(x)\right)\right|_{t=0} d H^{1}(x) \\
& =H^{1}(K \cap B)+\int_{K \cap A}\left\{f^{\prime}(r) \cos ^{2} \alpha(x)+\frac{f(r)}{r} \sin ^{2} \alpha(x)\right\} d H^{1}(x)
\end{aligned}
$$

by (3.20), (3.22), and (3.26).

Now we want to compute $e^{\prime}(0)$. Note that

$$
e(t)=\int_{B(0,1) \backslash K_{t}}\left|\nabla u_{t}\right|^{2}=\int_{\varphi_{t}(B(0,1) \backslash K)}\left|\nabla\left(u \circ \varphi_{t}^{-1}\right)\right|^{2},
$$

by the definitions (3.15) and (3.17). We split $\nabla\left(u \circ \varphi_{t}^{-1}\right)$ into its radial and tangential components and use (3.21). This yields

$$
\begin{aligned}
\frac{\partial}{\partial r}\left(u \circ \varphi_{t}^{-1}\right)\left(\varphi_{t}(x)\right) & =\frac{\partial u}{\partial r}(x) \frac{1}{g_{t}^{\prime}(r)}, \\
\frac{\partial}{\partial \tau}\left(u \circ \varphi_{t}^{-1}\right)\left(\varphi_{t}(x)\right) & =\frac{\partial u}{\partial \tau}(x) \frac{r}{g(r)} .
\end{aligned}
$$

Also, the Jacobian determinant of $\varphi_{t}$ at $x$ is the product $\frac{g_{t}^{\prime}(r) g_{t}(r)}{r}$. Altogether,

$$
\begin{aligned}
e(t) & =\int_{B(0,1) \backslash K}\left\{\left(\frac{\partial u}{\partial r} \frac{1}{g_{t}^{\prime}(r)}\right)^{2}+\left(\frac{\partial u}{\partial \tau} \frac{r}{g(r)}\right)^{2}\right\} \frac{g_{t}^{\prime}(r) g_{t}(r)}{r} d x \\
& =\int_{B(0,1) \backslash K}\left\{\left(\frac{\partial u}{\partial r}\right)^{2} \frac{g_{t}(r)}{r g_{t}^{\prime}(r)}+\left(\frac{\partial u}{\partial \tau}\right)^{2} \frac{r g_{t}^{\prime}(r)}{g(r)}\right\} d x .
\end{aligned}
$$

Note that

$$
\frac{g_{t}(r)}{r g_{t}^{\prime}(r)}=\frac{r+t f(r)}{r\left(1+t f^{\prime}(r)\right)}=\frac{1+t \frac{f(r)}{r}}{1+t f^{\prime}(r)}
$$


by (3.12). Recall from (3.13) that this is bounded and bounded from below, and its derivative with respect to $t$ at $t=0$ is $\frac{f(r)}{r}-f^{\prime}(r)$. Hence

$$
e^{\prime}(0)=\int_{B(0,1) \backslash K}\left\{\left(\frac{\partial u}{\partial r}\right)^{2}-\left(\frac{\partial u}{\partial \tau}\right)^{2}\right\}\left\{\frac{f(r)}{r}-f^{\prime}(r)\right\} .
$$

Next we use the specific formula (3.11) for $f$. As we could expect, we get no contribution from $B=B\left(0, r_{0}\right)$ because $\frac{f(r)}{r}=f^{\prime}(r)$ there (and our mapping $\varphi_{t}$ is conformal on $B$ ). On the remaining annulus $A$,

$$
\frac{f(r)}{r}-f^{\prime}(r)=\frac{r_{0}(1-r)}{r\left(1-r_{0}\right)}+\frac{r_{0}}{1-r_{0}}=\frac{r_{0}}{r\left(1-r_{0}\right)},
$$

and hence

$$
e^{\prime}(0)=\int_{A \backslash K}\left\{\left(\frac{\partial u}{\partial r}\right)^{2}-\left(\frac{\partial u}{\partial \tau}\right)^{2}\right\} \frac{r_{0}}{r\left(1-r_{0}\right)} .
$$

Now we know that $a^{\prime}(0)+e^{\prime}(0)$ exists, and (3.18) tells us that

$$
a^{\prime}(0)+e^{\prime}(0)=0 .
$$

We want to use (3.27) and (3.35), and let $r_{0}$ tend to $1^{-}$. Note that

$$
\lim _{r_{0} \rightarrow 1^{-}} e^{\prime}(0)=J_{\nu}-J_{\tau}
$$

(compare with (3.3) and (3.4), and recall for instance that $\nabla u$ is continuous on $A \backslash K$, and even bounded there, because $K$ is smooth on $A$ ). As for $a^{\prime}(0)$ and (3.27), notice that

$$
f^{\prime}(r) \cos ^{2} \alpha(x)+\frac{f(r)}{r} \sin ^{2} \alpha(x)=\frac{-r_{0}}{1-r_{0}} \cos ^{2} \alpha(x)+\frac{r_{0}(1-r)}{r\left(1-r_{0}\right)} \sin ^{2} \alpha(x)
$$

for $x \in A$, by (3.11). The second part stays bounded when $r_{0}$ tends to $1^{-}$, so it will not contribute in the limit. Hence (3.27) yields

$$
\begin{aligned}
\lim _{r_{0} \rightarrow 1^{-}} a^{\prime}(0) & =H^{1}(K \cap B(0,1))-\lim _{r_{0} \rightarrow 1^{-}} \frac{r_{0}}{1-r_{0}} \int_{K \cap A} \cos ^{2} \alpha(x) d H^{1}(x) \\
& =\ell(1)-\sum_{x \in K \cap \partial B(0,1)} \cos \alpha(x) .
\end{aligned}
$$

When we add up (3.37) and (3.39) and compare with (3.36), we get (3.6) with $r=1$. Proposition 3.5 follows.

\section{Simple energy bounds on harmonic extensions}

In this section we prove energy bounds on some harmonic extensions of functions defined on subarcs of the unit circle. These bounds will be used later when we construct 
competitors for our minimizer $(u, K)$. We start with the most basic case of harmonic extension.

Lemma 4.1. - Let $B=B(0,1)$ denote the unit disk, and let $u$ be a (real-valued) $C^{1}$ function on $\partial B$. Denote by $v$ the harmonic extension of $u$ to $B$. Then

$$
\int_{B}|\nabla v|^{2} \leqslant \int_{\partial B}\left(\frac{\partial u}{\partial \tau}\right)^{2} d H^{1}
$$

This is an easy computation with Fourier series. Let us identify $\mathbb{R}^{2}$ with $\mathbb{C}$ and write

$$
u\left(\mathrm{e}^{\mathrm{i} \theta}\right)=\sum_{k \in \mathbb{Z}} a_{k} \mathrm{e}^{\mathrm{i} k \theta} .
$$

The harmonic extension of $u$ to $B$ is given by

$$
v\left(r \mathrm{e}^{\mathrm{i} \theta}\right)=\sum_{k \in \mathbb{Z}} a_{k} r^{|k|} \mathrm{e}^{\mathrm{i} k \theta} .
$$

Then

$$
\begin{gathered}
\frac{\partial v}{\partial r}=\sum_{k \in \mathbb{Z}}|k| a_{k} r^{|k|-1} \mathrm{e}^{\mathrm{i} k \theta}, \\
\frac{\partial v}{\partial \tau}=\frac{1}{r} \frac{\partial v}{\partial \theta}=\sum_{k \in \mathbb{Z}} \mathrm{i} k a_{k} r^{|k|-1} \mathrm{e}^{\mathrm{i} k \theta}
\end{gathered}
$$

and, by Parseval,

$$
\int_{\partial B(0, r)}\left(\frac{\partial v}{\partial r}\right)^{2} d H^{1}=r \int_{0}^{2 \pi}\left(\frac{\partial v}{\partial r}\left(r \mathrm{e}^{\mathrm{i} \theta}\right)\right)^{2} d \theta=2 \pi \sum_{k \in \mathbb{Z}} k^{2}\left|a_{k}\right|^{2} r^{2|k|-1} .
$$

Similarly,

$$
\int_{\partial B(0, r)}\left(\frac{\partial v}{\partial \tau}\right)^{2} d H^{1}=2 \pi \sum_{k \in \mathbb{Z}} k^{2}\left|a_{k}\right|^{2} r^{2|k|-1} .
$$

Since $|\nabla v|^{2}=\left(\frac{\partial v}{\partial r}\right)^{2}+\left(\frac{\partial v}{\partial \tau}\right)^{2}$, we get that

$$
\begin{aligned}
\int_{B}|\nabla v|^{2} & =\int_{0}^{1} \int_{\partial B(0, r)}|\nabla v|^{2} d H^{1} d r=4 \pi \sum_{k \in \mathbb{Z}} k^{2}\left|a_{k}\right|^{2} \int_{0}^{1} r^{2|k|-1} d r \\
& =2 \pi \sum_{k \in \mathbb{Z}}|k|\left|a_{k}\right|^{2} \leqslant 2 \pi \sum_{k \in \mathbb{Z}} k^{2}\left|a_{k}\right|^{2}=\int_{\partial B}\left(\frac{\partial v}{\partial \tau}\right)^{2} d H^{1} .
\end{aligned}
$$

LEMMA 4.10. - Let $0<\alpha \leqslant 2$ be given, and set

$$
C(\alpha)=\left\{r \mathrm{e}^{\mathrm{i} \theta} ; 0<r<1 \text { and } 0<\theta<\pi \alpha\right\} .
$$


(a conic sector of aperture $\pi \alpha$ ) and $I(\alpha)=\left\{\mathrm{e}^{\mathrm{i} \theta} ; 0<\theta<\pi \alpha\right\}$ (the circular part of its boundary). Then for every $C^{1}$ function $u$ on $I(\alpha)$ such that $\int_{I(\alpha)}\left(\frac{\partial u}{\partial \tau}\right)^{2} d H^{1}<+\infty$ we can find a continuous function $v$ on $C(\alpha) \cup I(\alpha)$ which is harmonic in $C(\alpha)$ and such that

$$
\int_{C(\alpha)}|\nabla v|^{2} \leqslant \alpha \int_{I(\alpha)}\left(\frac{\partial u}{\partial \tau}\right)^{2} d H^{1} .
$$

We start with the special case when $\alpha=1$ and $C(\alpha)$ is the upper half disk. Extend $u$ to the whole circle by symmetry (that is, set $u\left(\mathrm{e}^{-\mathrm{i} \theta}\right)=u\left(\mathrm{e}^{\mathrm{i} \theta}\right)$ for $\left.0<\theta<\pi\right)$ and continuity (at 1 and -1 ). Call $v$ the harmonic extension to $B$ of the (extended) function $u$ on $\partial B$. Note that $v$ is also symmetric with respect to the first axis, and so

$$
\int_{C(1)}|\nabla v|^{2}=\frac{1}{2} \int_{B}|\nabla v|^{2} \leqslant \frac{1}{2} \int_{\partial B}\left(\frac{\partial u}{\partial \tau}\right)^{2} d H^{1}=\int_{I(1)}\left(\frac{\partial u}{\partial \tau}\right)^{2} d H^{1},
$$

by (the proof of) Lemma 4.1.

When $\alpha \neq 1$ we use the conformal mapping $\varphi: C(1) \rightarrow C(\alpha)$ defined by $\varphi\left(r \mathrm{e}^{\mathrm{i} \theta}\right)=$ $r^{\alpha} \mathrm{e}^{\mathrm{i} \alpha \theta}$. Let $u \in C^{1}(I(\alpha))$ be as in the statement, and set $u^{*}\left(\mathrm{e}^{\mathrm{i} \theta}\right)=u \circ \varphi\left(\mathrm{e}^{\mathrm{i} \theta}\right)=u\left(\mathrm{e}^{\mathrm{i} \alpha \theta}\right)$ for $0<\theta<\pi$. Then extend $u^{*}$ to $\mathrm{C}(1)$ as in the special case above. This gives a harmonic functions $v^{*}$ on $C(1)$. Finally set $v=v^{*} \circ \varphi^{-1}$ on $C(\alpha)$. Then

$$
\int_{C(\alpha)}|\nabla v|^{2}=\int_{C(1)}\left|\nabla v^{*}\right|^{2} \leqslant \int_{I(1)}\left(\frac{\partial u^{*}}{\partial \tau}\right)^{2} d H^{1}=\alpha \int_{I(\alpha)}\left(\frac{\partial u}{\partial \tau}\right)^{2} d H^{1}
$$

by conformal invariance of energy integral (or direct computation as in Section 3) and a linear change of variables on the circle. This proves the lemma.

We want to extend the result of Lemma 4.10 a little further, and for this the following definition will be convenient. Let $L \subset \mathbb{R}^{2}$ be closed, and let $V$ denote a connected component of $B \backslash L$. We shall say that $V$ is controlled by $C(\alpha)$ if there exists $\theta(V) \in \mathbb{R}$ such that $V \subset \mathrm{e}^{\mathrm{i} \theta(V)} C(\alpha)$ and $\partial V \backslash L$ is a single arc of $\partial B$ contained in $\mathrm{e}^{\mathrm{i} \theta(V)} I(\alpha)$. (see Fig. 2 for a typical example (where we could take $\theta(V)=0)$ ).

COROLlaRY 4.15. - Let $L \subset \mathbb{R}^{2}$ be closed, and suppose that for some $\alpha \in[0,2]$ every connected component of $B \backslash L$ is controlled by $C(\alpha)$. Let $u \in C^{1}(\partial B \backslash L)$ be such that

$$
J=\int_{\partial B \backslash L}\left(\frac{\partial u}{\partial \tau}\right)^{2} d H^{1}<+\infty .
$$

Then there is a continuous function $v$ on $\bar{B} \backslash L$ which is harmonic on $B \backslash L$, equal to $u$ on $\partial B \backslash L$, and such that

$$
\int_{B \backslash L}|\nabla v|^{2} \leqslant \alpha J
$$




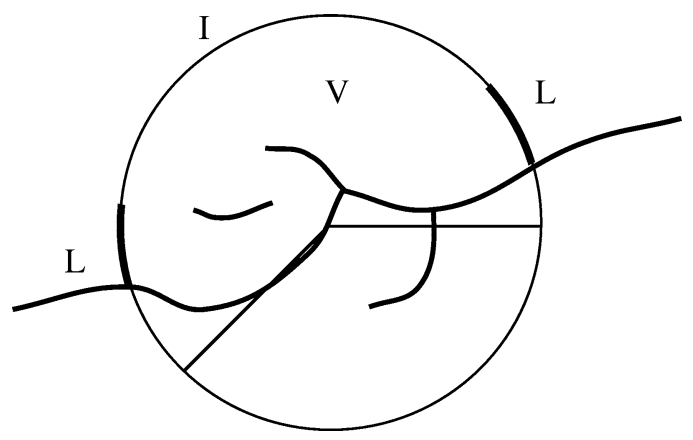

Fig. 2.

We want to construct the extension $v$ component of $B \backslash L$ by component. So let $V$ denote a connected component of $B \backslash L$, and let $\theta(V) \in \mathbb{R}$ be such that $V$ is contained in $\mathrm{e}^{\mathrm{i} \theta(V)} C(\alpha)$. Set $I(V)=\partial V \backslash L$; by assumption $I(V)$ is an arc of circle contained in $\mathrm{e}^{\mathrm{i} \theta(V)} I(\alpha)$, where $I(\alpha)=\left\{\mathrm{e}^{\mathrm{i} \theta} ; 0<\theta<\pi \alpha\right\}$. Let $u_{V}$ be an extension of $\left.u\right|_{I(V)}$ to $\mathrm{e}^{\mathrm{i} \theta(V)} I(\alpha)$ such that $\int_{\mathrm{e}^{\mathrm{i} \theta(V)} I(\alpha)}\left(\frac{\partial u_{V}}{\partial \tau}\right)^{2} d H^{1}=\int_{I(V)}\left(\frac{\partial u}{\partial \tau}\right)^{2} d H^{1}$. Then use Lemma 4.10 to find an extension $v_{V}$ of $u_{V}$ on $\mathrm{e}^{\mathrm{i} \theta(V)}[C(\alpha) \cup I(\alpha)]$. Then

$$
\int_{V}\left|\nabla v_{V}\right|^{2} \leqslant \int_{\mathrm{e}^{\mathrm{i} \theta(V)} C(\alpha)}\left|\nabla v_{V}\right|^{2} \leqslant \alpha \int_{\mathrm{e}^{\mathrm{i} \theta(V)} I(\alpha)}\left(\frac{\partial u_{V}}{\partial \tau}\right)^{2} d H^{1}=\alpha \int_{I(V)}\left(\frac{\partial u}{\partial \tau}\right)^{2} d H^{1} .
$$

Now define $v$ on $B \backslash L$ simply by taking $v=v_{V}$ on $V$ for each component. There is no ambiguity because the components are disjoint, $v$ is harmonic on $B \backslash L$ because each $v_{V}$ is harmonic on $V$, and $v$ is continuous also at points of $\partial B \backslash L$ because if $x \in \partial B \backslash L$, the connected component of $x$ in $\partial B \backslash L$ is an open interval that contains $x$ and is contained in a single $I(V)$. Finally (4.17) follows from (4.18) because the intervals $I(V)$ are disjoint.

\section{Proof of our monotonicity estimates}

We are now ready to start the proof of the various lower bounds on $F^{\prime}(r), r \in \mathcal{R}$, announced in Section 2.

Let us simplify the notation first. For the same homogeneity reasons as for Proposition 3.5 , it is enough to prove all these estimates when $r=1$. So let us assume that $1 \in \mathcal{R}$ and try to prove lower bounds for $F^{\prime}(1)$. Set $B=B(0,1), E=E(1)=\int_{B \backslash K}|\nabla u|^{2}, \ell=$ $\ell(1)=H^{1}(K \cap B)$, and call $N=N(1)$ the number of points in $K \cap \partial B$. Recall from (3.8) and (3.9) that

$$
\begin{gathered}
\frac{1}{2} F^{\prime}(1) \geqslant \frac{3}{2} J_{\tau}+\frac{1}{2} J_{v}+N-E-\ell ; \\
F^{\prime}(1) \geqslant J_{\tau}+3 J_{v}-2 E,
\end{gathered}
$$

where $J_{\tau}$ and $J_{v}$ are still as in (3.3) and (3.4). 
Most of our estimates will come from upper bounds on $E+\ell$, which will be obtained by comparing our minimizer $(u, K)$ with various competitors $(v, L)$. Maybe we should say now that all our competitors $(v, L)$ will coincide with $(u, K)$ outside of $\bar{B}$, so that the results of Section 2 only use the minimality of the pair $(u, K)$ in a neighborhood of $\bar{B}$. See Section 6 for relevant definitions.

Another common feature of our pairs $(v, L)$ will be that in all cases,

$$
K \cap \partial B \subset L \cap \partial B
$$

and

$$
\left\{\begin{array}{l}
\text { if } I_{1}, I_{2} \text { are different connected components of } \partial B \backslash L, \\
\text { then they lie in different components of } \bar{B} \backslash L \text {. }
\end{array}\right.
$$

In fact, $\partial B \backslash L$ will always be a finite union of open $\operatorname{arcs}$ of $\partial B$, and (5.4) says that $L$ separates them from each other in $\bar{B}$. Let us already check now that (5.3) and (5.4) automatically imply the topological condition (1.4).

Let us even check that if $x, y \in \mathbb{R}^{2} \backslash(\bar{B} \cup K)$ lie in different components of $\mathbb{R}^{2} \backslash K$, then they lie in different components of $\mathbb{R}^{2} \backslash L$ as well. Let us proceed by contradiction and assume instead that there is a simple arc $\gamma$ in $\mathbb{R}^{2} \backslash L$ that goes from $x$ to $y$. This arc meets $K$ because $x, y$ lie in different components of $\mathbb{R}^{2} \backslash K$. Since $K$ and $L$ coincide out of $\bar{B}, \gamma$ meets $\bar{B}$.

Call $x_{1}$ the first point of $\gamma \cap \partial B$ that we meet when we start from $x$ and run along $\gamma$. Then $x_{1} \in \partial B \backslash L$, because $\gamma$ does not meet $L$. Hence $x_{1} \in \partial B \backslash K$, by (5.3). Moreover, $x_{1} \in U(x)$, the component of $x$ in $\mathbb{R}^{2} \backslash K$, because the arc of $\gamma$ between $x$ anf $x_{1}$ lies in $\mathbb{R}^{2} \backslash(L \cup \bar{B})=\mathbb{R}^{2} \backslash(K \cup \bar{B})$.

Similary call $y_{1}$ the first point of $\gamma \cap \partial B$ that we meet when we start from $y$ and run along $\gamma$ backwards. Then $y \in \partial B \cap U(y)$ (the component of $y$ in $\mathbb{R}^{2} \backslash K$ ) for similar reasons. In particular $y_{1}$ does not lie in $U(x)$.

Next call $y_{2}$ the first point of $\partial B \backslash U(x)$ that we meet when we start from $x_{1}$ and run along $\gamma$ in the direction of $y_{1}$ and $y$. Such a point exists because $\partial B \backslash U(x)$ is closed and contains $y_{1}$.

Note that $y_{2} \in \partial B \backslash L \subset \partial B \backslash K$, because $\gamma \subset \mathbb{R}^{2} \backslash L$ and by (5.3). Let us now start from $y_{2}$ and run along $\gamma$ backwards (in the direction of $x_{1}$ ). We start in $\mathbb{R}^{2} \backslash K$, and in a component $U\left(y_{2}\right) \neq U(x)$ (because $\left.y_{2} \notin U(x)\right)$. We do not meet $\partial B$ immediately, because otherwise $y_{2}$ would not be the first point of $\gamma \in \partial B \backslash U(x)$ after $x_{1}$. So there is a first point $x_{2}$ in $\gamma \cap \partial B$ (when we start from $y_{2}$ and run along $\gamma$ backwards).

This point is between $x_{1}$ and $y_{2}$, and it lies in $U(x)$ by definition of $y_{2}$. The open subarc $\gamma_{2}$ of $\gamma$ between $x_{2}$ and $y_{2}$ does not meet $\partial B$, hence it lies in $\mathbb{R}^{2} \backslash \bar{B}$ or in $B$. The first option is impossible, because $\gamma$ does not meet $K \backslash \bar{B}=L \backslash \bar{B}$ and $x_{2}, y_{2}$ lie in different components of $\mathbb{R}^{2} \backslash K$. The second option also is impossible, because (5.3) tells us that $x_{2}, y_{2}$ lie in different components of $\partial B \backslash L$ (they lie in $\partial B \backslash L$ because $\gamma$ does not meet $L$ ), and then (5.4) forces $\gamma_{2}$ to meet $L$.

This contradiction completes our verification of (1.4) when (5.3) and (5.4) hold.

Proof of Proposition 2.7. - All we need to check here is that

$$
F^{\prime}(1) \geqslant \int_{\partial B}|\nabla u|^{2}=J_{\nu}+J_{\tau} \text { when } K \cap \partial B=\emptyset .
$$


For this we set $L=K \backslash B$, keep $v=u$ out of $B \cup K$, and let $\left.v\right|_{B}$ be the harmonic extension of $\left.u\right|_{\partial B}$. It is easy to see that $(v, L)$ is a competitor for $(u, K)$. Then (1.6) holds and we get that

$$
E+\ell=H^{1}(K \cap B)+\int_{B \backslash K}|\nabla u|^{2} \leqslant \int_{B}|\nabla v|^{2} \leqslant \int_{\partial B}\left(\frac{\partial u}{\partial \tau}\right)^{2} d H^{1}=J_{\tau},
$$

by Lemma 4.1 and the definition (3.3). Now (5.5) follows from (5.1).

Proof of Proposition 2.13. - It will be convenient, here and in the rest of this section, to denote by $\pi \omega, 0<\omega \leqslant 2$, the length of the largest component of $\partial B \backslash K$. We want to check that

$$
F^{\prime}(1) \geqslant \min (1,3-2 \omega) \int_{\partial B \backslash K}|\nabla u|^{2} .
$$

We do not need to assume that $\omega \leqslant 3 / 2$ as we did in Proposition 2.13, but in the other cases (5.7) will be hard to use anyway.

Set $L=X \cup(K \backslash B)$, where $X$ denotes the union of the $N$ radial segments [0, $x], x \in$ $K \cap \partial B$. Note that $L$ satisfies our conditions (5.3) and (5.4), so there will be no trouble with (1.4). We keep $v=u$ out of $B \cup K$ and, to define $v$ on $B \backslash L$, we apply Corollary 4.15 (or directly Lemma 4.10 to each component of $B \backslash L$ ). We can do this with $\alpha=\omega$. This gives a function $v$ such that

$$
\int_{B \backslash L}|\nabla v|^{2} \leqslant \omega \int_{\partial B \backslash K}\left(\frac{\partial u}{\partial \tau}\right)^{2} d H^{1}=\omega J_{\tau} .
$$

It is easy to check that $(v, L)$ is a competitor for $(u, K)$, so (1.6) holds and

$$
E+\ell \leqslant H^{1}(L \cap B)+\int_{B \backslash L}|\nabla v|^{2} \leqslant H^{1}(X)+\omega J_{\tau}=N+\omega J_{\tau} .
$$

Then (5.1) says that

$$
\frac{1}{2} F^{\prime}(1) \geqslant \frac{3}{2} J_{\tau}+\frac{1}{2} J_{v}-\omega J_{\tau} \geqslant \frac{1}{2} \min (1,3-2 \omega)\left(J_{\tau}+J_{v}\right),
$$

and (5.7) follows because $\int_{\partial B \backslash K}|\nabla u|^{2}=J_{\tau}+J_{\nu}$.

Proof of Proposition 2.12. - We start with the case when

$$
N \geqslant 4 \text { and } \omega \geqslant 1.4 \text {. }
$$

Denote by $I$ the longest component of $\partial B \backslash K$. Thus $H^{1}(I)=\pi \omega$ and $H^{1}(\partial B \backslash I) \leqslant$ $\frac{6 \pi}{10}<1.9$. Let $I^{\prime}$ denote an arc of $\partial B$ that contains $K \cap \partial B$ and such that $H^{1}\left(I^{\prime}\right)=1.9$ (see Fig. 3). 


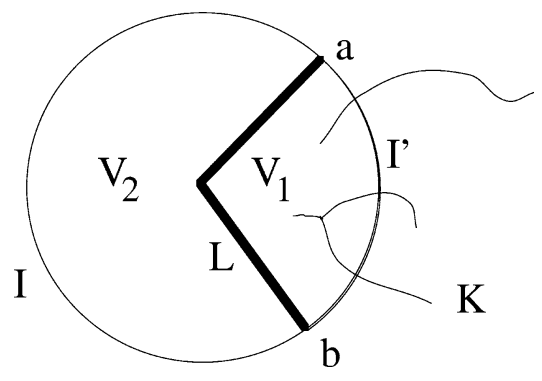

Fig. 3.

Call $a, b$ the extremities of $I^{\prime}$, and set

$$
L=(K \backslash B) \cup I^{\prime} \cup[0, a] \cup[0, b]
$$

Thus $H^{1}(L \cap \bar{B})=3.9$ and once again $L$ satisfies (5.3) and (5.4).

The set $B \backslash L$ has two connected components, a smaller one $V_{1}$ which is entirely surrounded by $L$ and a larger one $V_{2}$. We keep $v=u$ out of $B \cup L$, set $v=0$ on $V_{1}$, and to define $v$ on $V_{2}$ we apply Corollary 4.15 or Lemma 4.10 with $\alpha=2-H^{1}\left(I^{\prime}\right) / \pi \leqslant 1.4$. We get an extension of $\left.u\right|_{\partial B \backslash I^{\prime}}$ to $V_{2}$ such that $\int_{V_{2}}|\nabla v|^{2} \leqslant 1.4 J_{\tau}$.

This defines a competitor $(v, L)$ for $(u, K)$, and (1.6) (applied to a ball slightly larger than $B$ ) yields

$$
E+\ell \leqslant H^{1}(L \cap \bar{B})+\int_{B \backslash L}|\nabla v|^{2} \leqslant 3.9+1.4 J_{\tau} .
$$

Then (5.1) says that

$$
\frac{1}{2} F^{\prime}(1) \geqslant \frac{3}{2} J_{\tau}+\frac{1}{2} J_{\nu}+N-3.9-1.4 J_{\tau} \geqslant \frac{1}{10}
$$

because $N \geqslant 4$. This proves the desired estimate when (5.11) holds. The other case is when

$$
N \geqslant 4 \text { and } \omega<1.4
$$

We may as well assume that

$$
\int_{\partial B \backslash K}|\nabla u|^{2} \leqslant 10^{-2},
$$

because otherwise we can apply (5.7) and get that $F^{\prime}(1) \geqslant \frac{1}{10} \int_{\partial B \backslash K}|\nabla u|^{2} \geqslant 10^{-3}$ as needed.

We want to replace $K \cap B$ with something shorter than $N$, and for this and similar estimates later the following definition will be useful. 


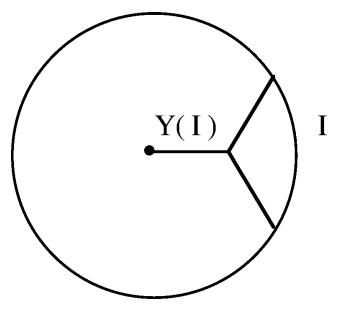

(a)

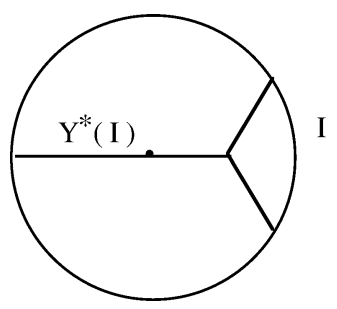

(b)

Fig. 4.

Definition 5.17. - Let I be an interval of $\partial B$, with $H^{1}(I)<2 \pi / 3$. Call $a, b$ the extremities of $I$. The fork based on $I$ is the set

$$
Y(I)=[0, x] \cup[x, a] \cup[x, b],
$$

where the center $x \in B$ is chosen so that the 3 segments in (5.18) make $120^{\circ}$ angles at $x$. (See Fig. 4(a).) The extended fork based on I is the set

$$
Y^{*}(I)=Y(I) \cup\left[\frac{-x}{|x|}, 0\right]
$$

where we add the radius opposite to $[0, x]$. (See Fig. 4(b).)

The point of the fork is that it is the shortest connected set that contains $0, a$, and $b$, but we shall not need to know this.

LEMMA 5.20. - Set $f(\alpha)=2 \sin \left(\frac{\alpha \pi}{2}+\frac{\pi}{6}\right)$ for $0<\alpha<\frac{2}{3}$. Then

$$
H^{1}(Y(I))=f(\alpha) \quad \text { when } H^{1}(I)=\alpha \pi .
$$

We may assume that the center $x$ lies on the first axis, and even that $x \geqslant 0$, as in Fig. 5 . Then the endpoints of $I$ are $\mathrm{e}^{ \pm \mathrm{i} \frac{\alpha \pi}{2}}$. Hence $H^{1}([x, a])=\frac{2}{\sqrt{3}} \sin \frac{\alpha \pi}{2}$ and

$$
x=\cos \frac{\alpha \pi}{2}-\frac{1}{2} H^{1}([x, a])=\cos \frac{\alpha \pi}{2}-\frac{1}{\sqrt{3}} \sin \frac{\alpha \pi}{2} .
$$

Now

$$
\begin{aligned}
H^{1}(Y(I)) & =x+2 H^{1}([x, a])=\cos \frac{\alpha \pi}{2}+\frac{3}{\sqrt{3}} \sin \frac{\alpha \pi}{2} \\
& =2\left\{\frac{1}{2} \cos \frac{\alpha \pi}{2}+\frac{\sqrt{3}}{2} \sin \frac{\alpha \pi}{2}\right\}=f(\alpha),
\end{aligned}
$$

as needed for the lemma.

We shall need to know that

$$
f(\alpha) \text { is an increasing function of } \alpha \in\left(0, \frac{2}{3}\right)
$$




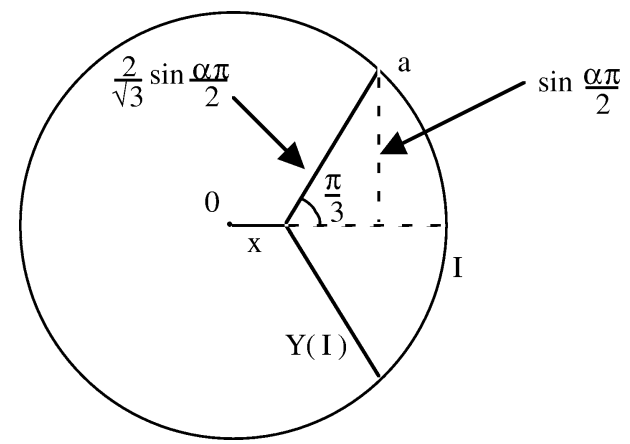

Fig. 5.

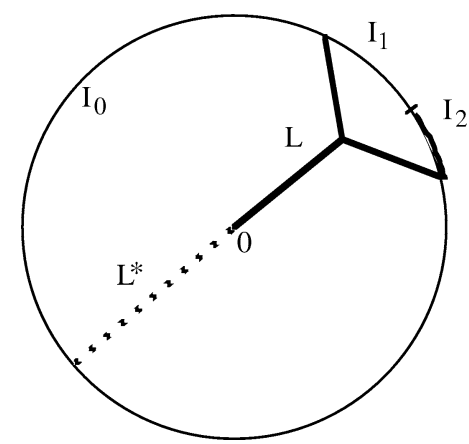

Fig. 6. $L \cap \bar{B}$ and $L^{*} \cap \bar{B}$.

and

$$
\begin{aligned}
& f(0.5)<1.95, \quad f(0.4)<1.83, \quad f(0.268)<1.6249, \\
& f(0.251)<1.59, \quad f(0.22)<1.53, \quad \text { and } \quad f(0.2)<1.5 .
\end{aligned}
$$

This set of values is a little arbitrary, the estimates in (5.25) are not very sharp, and we do not propose any proof other than checking with a calculator if the reader wishes.

Let us return to our case when (5.15) and (5.16) hold and try to construct a new set $L$. Call $I$ the shortest component of $\partial B \backslash K$. Then $H^{1}(I) \leqslant \frac{\pi}{2}$, because $N \geqslant 4$. Write $K \cap \partial B=\left\{a_{1}, a_{2}, \ldots, a_{N}\right\}$, where the indices are chosen so that $a_{1}$ and $a_{2}$ are the extremities of $I$. Then set

$$
L=(K \backslash B) \cup Y(I) \cup\left[0, a_{3}\right] \cup \cdots \cup\left[0, a_{N}\right] .
$$

Note that (5.3) and (5.4) are satisfied as usual, and

$$
H^{1}(L \cap B) \leqslant f\left(\frac{H^{1}(I)}{\pi}\right)+N-2 \leqslant f(0.5)+N-2 \leqslant N-\frac{5}{100},
$$

by (5.24) and (5.25). Let us define $v$ on $B \backslash L$ by a brutal application of Corollary 4.15 with $\alpha \leqslant 2$. As usual we keep $v=u$ out of $B,(v, L)$ is a competitor for $(u, K)$, and (1.6) yields

$$
E+\ell \leqslant H^{1}(L \cap B)+\int_{B \backslash L}|\nabla v|^{2} \leqslant 2 J_{\tau}+N-\frac{5}{100} \leqslant N-\frac{3}{100},
$$

by (5.16). Then (5.1) says that $\frac{1}{2} F^{\prime}(1) \geqslant \frac{3}{100}$, as needed for Proposition 2.12.

The rest of this section will be devoted to the proof of Proposition 2.9. Let us already check that

$$
\text { we can assume that } \int_{\partial B \backslash K}|\nabla u|^{2} \leqslant 10 \text {. }
$$

Indeed, if this is not the case we note that $E+\ell \leqslant 2 \pi$ by (2.4), and (5.1) yields 


$$
\begin{aligned}
\frac{1}{2} F^{\prime}(1) & \geqslant \frac{3}{2} J_{\tau}+\frac{1}{2} J_{v}+N-E-\ell \geqslant \frac{1}{2} \int_{\partial B \backslash K}|\nabla u|^{2}+2-2 \pi \\
& \geqslant \frac{1}{20} \int_{\partial B \backslash K}|\nabla u|^{2}+6.5-2 \pi,
\end{aligned}
$$

from which (2.11) follows easily.

Now we can easily settle the case when $N \geqslant 4$. Indeed, (2.11) follows from Proposition 2.12, because of (5.29).

We continue our study with the case when $N=3$, and even

$$
N=3 \text { and } \omega \geqslant 1.6
$$

Let us construct a first competitor $(v, L)$. Call $I_{0}$ the largest component of $\partial B \backslash K$ and set $I=\partial B \backslash I_{0}$. Thus $H^{1}(I) \leqslant \frac{4 \pi}{10}$ and $H^{1}(Y(I)) \leqslant f(0.4) \leqslant 1.83$ by (5.24) and (5.25).

Call $I_{1}$ and $I_{2}$ the two components of $I \backslash K$ (i.e., the two shortest components of $\partial B \backslash K)$. We may as well assume that $H^{1}\left(I_{2}\right) \leqslant H^{1}\left(I_{1}\right)$. Then $H^{1}\left(I_{2}\right) \leqslant \frac{1}{2}(2 \pi-$ $\left.H^{1}\left(I_{0}\right)\right)=\pi-\frac{\omega \pi}{2}$. We take

$$
L=(K \backslash B) \cup Y(I) \cup \overline{I_{2}}
$$

(see Fig. 6). The point of adding $\bar{I}_{2}$ here is to make sure that (5.3) and (5.4) hold.

As usual we can define $v$ on $B \backslash L$ with the help of Corollary 4.15 , but we have to take $\alpha=2$. Then (1.6) yields

$$
\begin{aligned}
E+\ell & \leqslant H^{1}(L \cap \bar{B})+\int_{B \backslash L}|\nabla v|^{2} \leqslant H^{1}(Y(I))+H^{1}\left(I_{2}\right)+2 J_{\tau} \\
& \leqslant 1.83+\pi-\frac{\omega \pi}{2}+2 J_{\tau} \leqslant 1.83+\frac{2 \pi}{10}+2 J_{\tau}
\end{aligned}
$$

(because $\omega \geqslant 1.6)$. We do not want to apply (5.1) immedialety; instead we introduce another competitor $\left(v^{*}, L^{*}\right)$ which may be better if $J_{\tau}>1$. Take $L^{*}=(K \backslash B) \cup Y^{*}(I) \cup$ $\bar{I}_{2}$. This is similar to (5.32), but we replaced the fork $Y(I)$ with the extended fork $Y^{*}(I)$. Thus $H^{1}\left(L^{*} \cap \bar{B}\right)=H^{1}(L \cap \bar{B})+1$. The point of taking $Y^{*}(I)$ is that now all the components of $B \backslash L^{*}$ are contained in half-disks, and we can use Corollary 4.15 with $\alpha=1$ to define $v^{*}$ on $B \backslash L^{*}$. The same argument as for (5.33) now yields

$$
\begin{aligned}
E+\ell & \leqslant H^{1}\left(L^{*} \cap \bar{B}\right)+\int_{B \backslash L^{*}}\left|\nabla v^{*}\right|^{2} \leqslant H^{1}(L \cap \bar{B})+1+J_{\tau} \\
& \leqslant 2.83+\frac{2 \pi}{10}+J_{\tau} .
\end{aligned}
$$

We average (5.33) and (5.34) and get that

$$
E+\ell \leqslant 2.33+\frac{2 \pi}{10}+\frac{3}{2} J_{\tau} \leqslant 2.96+\frac{3}{2} J_{\tau}
$$


and then (5.1) says that

$$
\frac{1}{2} F^{\prime}(1) \geqslant \frac{3}{2} J_{\tau}+3-E-\ell \geqslant \frac{4}{100} \geqslant \frac{4}{1000} \int_{\partial B \backslash K}|\nabla u|^{2},
$$

because of (5.29).

Our next case is when

$$
N=3 \quad \text { and } \quad 1.49 \leqslant \omega \leqslant 1.6 .
$$

Call $I_{0}, I_{1}, I_{2}$ the components of $\partial B \backslash K$, with $H^{1}\left(I_{0}\right)>H^{1}\left(I_{1}\right) \geqslant H^{1}\left(I_{2}\right)$. Let $I$ be a closed interval of $\partial B$ which contains $I_{1} \cup I_{2}$ and whose length is $H^{1}(I)=\frac{51 \pi}{100}$. Denote by $a, b$ the extremities of $I$, and set

$$
L=(K \backslash B) \cup\left(I \backslash I_{1}\right) \cup[0, a] \cup[0, b],
$$

as suggested by Fig. 7. Then (5.3) and (5.4) hold, and

$$
\begin{aligned}
H^{1}(L \cap \bar{B}) & =2+H^{1}(I)-H^{1}\left(I_{1}\right) \leqslant 2+\frac{51 \pi}{100}-\frac{1}{2}(2 \pi-\omega \pi) \\
& \leqslant 2+\frac{51 \pi}{100}-\frac{2 \pi}{10}<2.98 .
\end{aligned}
$$

We can construct $v$ on $B \backslash L$ by applying Corollary 4.15 with $\alpha=1.49$. Then the usual comparison argument yields

$$
E+\ell \leqslant H^{1}(L \cap \bar{B})+1.49 J_{\tau} \leqslant 2.98+1.49 J_{\tau},
$$

and then (5.1) says that

$$
\frac{1}{2} F^{\prime}(1) \geqslant \frac{3}{2} J_{\tau}+3-E-\ell \geqslant \frac{2}{100},
$$

from which (2.11) follows because of (5.29).

Our next case is when $N=3$ and $\omega \leqslant 1.49$, but then (2.11) follows readily from Proposition 2.13.

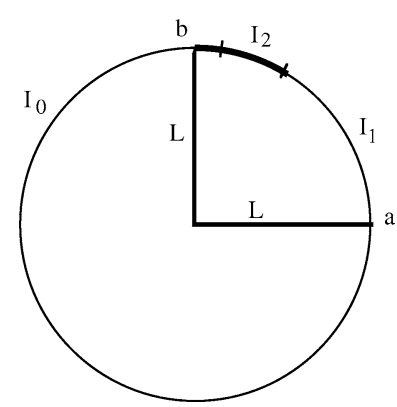

Fig. 7.
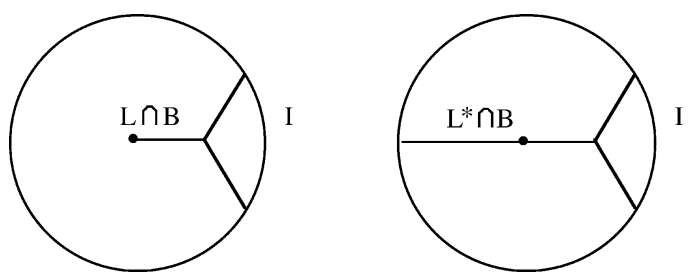

Fig. 8 . 
Thus we are left with the case when $N=2$. We can assume that $\omega \geqslant 1.49$ because otherwise (2.11) follows from Proposition 2.13.

Let us first try to get results without using our topological assumption (2.10). Let $I$ denote the shortest component of $\partial B \backslash K$, and let us try a first competitor with

$$
L=(K \backslash B) \cup Y(I),
$$

where $Y(I)$ still denotes the fork based on $I$, as in Proposition 5.17. See Fig. 8, and note that $H^{1}(I)<2 \pi / 3$ because $\omega \geqslant 1.49$.

This is similar to what we did when $N=3$, a little simpler. We can apply Corollary 4.15 with $\alpha=2$ and get an extension $v$ such that $\int_{B \backslash L}|\nabla v|^{2} \leqslant 2 J_{\tau}$. Then (1.6) yields

$$
E+\ell \leqslant H^{1}(Y(I))+\int_{B \backslash L}|\nabla v|^{2} \leqslant f(2-\omega)+2 J_{\tau},
$$

by Lemma 5.20, and (5.1) yields

$$
\frac{1}{2} F^{\prime}(1) \geqslant \frac{3}{2} J_{\tau}+\frac{1}{2} J_{v}+2-E-\ell \geqslant-\frac{1}{2} J_{\tau}+\frac{1}{2} J_{v}+2-f(2-\omega) .
$$

When $J_{\tau}>1$ it is more advantagous to use $L^{*}=(K \backslash B) \cup Y^{*}(I)$, with the extended fork $Y^{*}(I)$, instead of $L$.

This increases the length by 1 , but now the components of $B \backslash L^{*}$ are contained in half-disks and we can apply Corollary 4.15 with $\alpha=1$. The same computations as for (5.43) and (5.44) yield

$$
\begin{gathered}
E+\ell \leqslant 1+f(2-\omega)+J_{\tau}, \\
\frac{1}{2} F^{\prime}(1) \geqslant \frac{1}{2} J_{\tau}+\frac{1}{2} J_{v}+1-f(2-\omega)
\end{gathered}
$$

and, when we average with (5.44),

$$
\frac{1}{2} F^{\prime}(1) \geqslant \frac{1}{2} J_{v}+\frac{3}{2}-f(2-\omega) .
$$

These are the estimates that we can get without using (2.10). For the next one we have to use (2.10) and an argument of Bonnet.

LEMMA 5.48. - If $N=2$ and (2.10) holds,

$$
E \leqslant \omega J_{\tau}^{1 / 2} J_{v}^{1 / 2} .
$$

The proof is directly out of [4]; we review it here for the convenience of the reader but refer to [4] for (some) more details. First,

$$
E=\int_{\partial B \backslash K} u \frac{\partial u}{\partial v} d H^{1},
$$



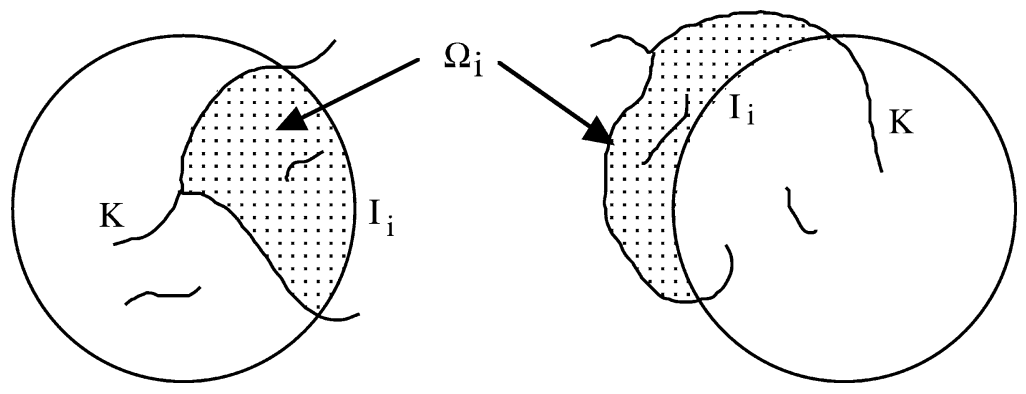

Fig. 9.

by a standard computation that uses Green's formula, the fact that $u$ is harmonic on $\mathbb{R}^{2} \backslash K$, and the Neumann condition $\frac{\partial u}{\partial n}=0$ on $K \cap B$. Next call $I_{1}, I_{2}$ the two components of $\partial K \backslash B$. Then

$$
\int_{I_{i}} \frac{\partial u}{\partial v}=0 \quad \text { for } i=1,2 .
$$

This uses the Neumann condition $\frac{\partial u}{\partial n}=0$ on $K$ again, plus the fact that since (2.10) tells us that the two extremities of $I_{i}$ lie on the same component of $K$, we can find a bounded domain $\Omega_{i} \subset \mathbb{R}^{2} \backslash K$ whose boundary is composed of $I_{i}$, plus a subset of $K$ where $\frac{\partial u}{\partial v}=0$. See Fig. 9 for a hint. So that, by Green's formula,

$$
0=\int_{\Omega_{i}} \Delta u=\int_{\partial \Omega_{i}} \frac{\partial u}{\partial n}= \pm \int_{I_{i}} \frac{\partial u}{\partial n}= \pm \int_{I_{i}} \frac{\partial u}{\partial v} .
$$

Next we deduce from (5.50) and (5.51) that

$$
E=\int_{\partial B \backslash K} u \frac{\partial u}{\partial v}=\sum_{i=1}^{2} \int_{I_{i}} u \frac{\partial u}{\partial v}=\sum_{i=1}^{2} \int_{I_{i}}\left(u-m_{i}\right) \frac{\partial u}{\partial v},
$$

where $m_{i}$ denotes the mean value of $u$ on $I_{i}$. Then for each $\lambda>0$

$$
\begin{aligned}
\int_{I_{i}}\left(u-m_{i}\right) \frac{\partial u}{\partial v} & \leqslant\left\{\int_{I_{i}}\left(u-m_{i}\right)^{2}\right\}^{1 / 2}\left\{\int_{I_{i}}\left(\frac{\partial u}{\partial v}\right)^{2}\right\}^{1 / 2} \\
& \leqslant \frac{\lambda}{2} \int_{I_{i}}\left(u-m_{i}\right)^{2}+\frac{1}{2 \lambda} \int_{I_{i}}\left(\frac{\partial u}{\partial v}\right)^{2} .
\end{aligned}
$$

Now we use an inequality of Wirtinger on $I_{i}$ (see [14]), namely

$$
\int_{I_{i}}\left(u-m_{i}\right)^{2} \leqslant\left(\frac{H^{1}\left(I_{i}\right)}{\pi}\right)^{2} \int_{I_{i}}\left(\frac{\partial u}{\partial \tau}\right)^{2} \leqslant \omega^{2} \int_{I_{i}}\left(\frac{\partial u}{\partial \tau}\right)^{2} .
$$


Altogether

$$
E \leqslant \sum_{i=1}^{2}\left\{\frac{\lambda}{2} \omega^{2} \int_{I_{i}}\left(\frac{\partial u}{\partial \tau}\right)^{2}+\frac{1}{2 \lambda} \int_{I_{i}}\left(\frac{\partial u}{\partial \nu}\right)^{2}\right\}=\frac{\lambda}{2} \omega^{2} J_{\tau}+\frac{1}{2 \lambda} J_{\nu} .
$$

We choose $\lambda=\omega^{-1} J_{\tau}^{-1 / 2} J_{v}^{1 / 2}$ and get (5.49).

We may now return to the proof of Proposition 2.9 when $N=2$. Exceptionally we use (5.2) and get that

$$
F^{\prime}(1) \geqslant J_{\tau}+3 J_{v}-2 E \geqslant J_{\tau}+3 J_{v}-2 \omega J_{\tau}^{1 / 2} J_{v}^{1 / 2}=x^{2}+3 y^{2}-2 \omega x y,
$$

where we set $x=J_{\tau}^{1 / 2}$ and $y=J_{v}^{1 / 2}$.

With the same notations, (5.44) and (5.47) say that

$$
\begin{gathered}
F^{\prime}(1) \geqslant-x^{2}+y^{2}+4-2 f(2-\omega), \\
F^{\prime}(1) \geqslant y^{2}+3-2 f(2-\omega) .
\end{gathered}
$$

Nothing clever will happen in the rest of the argument; we shall just distinguish cases, depending on the values of $\omega, x$, and $y$, and use (5.57)-(5.59) to prove that $F^{\prime}(1) \geqslant 10^{-9}$ in all cases. Of course (2.11) will follow, because of (5.29).

Recall that we can assume that $\omega \geqslant 1.49$ because of Proposition 2.13. We start with the case when

$$
1.49 \leqslant \omega \leqslant \omega_{0}, \quad \text { with } \omega_{0}=1.732 .
$$

We shall see very soon why we chose $\omega_{0}$ just a little smaller that $\sqrt{3}$. Note that $\frac{\omega_{0}^{2}}{3} x^{2}+3 y^{2}-2 \omega_{0} x y \geqslant 0$, because it is a square. Hence (5.57) says that

$$
F^{\prime}(1) \geqslant x^{2}+3 y^{2}-2 \omega x y \geqslant x^{2}+3 y^{2}-2 \omega_{0} x y \geqslant\left(1-\frac{\omega_{0}^{2}}{3}\right) x^{2} \geqslant 10^{-5} x^{2} .
$$

If $x^{2} \geqslant 10^{-4}$, then $F^{\prime}(1) \geqslant 10^{-9}$ and we are happy. Otherwise we can use (5.58) to get that

$$
F^{\prime}(1) \geqslant-x^{2}+y^{2}+4-2 f(2-\omega) \geqslant 4-2 f(0.51)-10^{-4}>10^{-4}
$$

by (5.24), (5.60), and an estimate for $f(0.51)$ which was not recorded in (5.25) but is very easy to believe. This settles our first case; we may now assume that $\omega \geqslant \omega_{0}$. Then

$$
f(2-\omega) \leqslant f\left(2-\omega_{0}\right)=f(0.268)<1.6249
$$

by (5.24) and (5.25). (It looks here that we need very sharp estimates, but this is largely a joke.) Our next case is when

$$
\omega \geqslant \omega_{0} \quad \text { and } \quad y^{2} \geqslant \frac{1}{4}
$$


Then (5.59) says that

$$
F^{\prime}(1) \geqslant y^{2}+3-2 f(2-\omega) \geqslant 3.25-2 f(2-\omega)>10^{-4},
$$

which is enough. Similary, if

$$
\omega \geqslant \omega_{0} \quad \text { and } \quad x^{2} \leqslant y^{2}+\frac{3}{4}
$$

(5.58) says that

$$
F^{\prime}(1) \geqslant-x^{2}+y^{2}+4-2 f(2-\omega) \geqslant 3.25-2 f(2-\omega) \geqslant 10^{-4}
$$

and we are equally happy.

Next set $\omega_{1}=\frac{7}{4}-10^{-3}=1.749$ and study the case when

$$
\omega_{0} \leqslant \omega \leqslant \omega_{1} \text {, but (5.64) and (5.66) fail. }
$$

In particular $y^{2}<\frac{1}{4}$ and $x^{2}>y^{2}+\frac{3}{4}$, hence $x^{2}>4 y^{2}$. Since (5.58) and (5.59) failed us we return to (5.57). Set

$$
h_{1}(x, y)=\left(1-10^{-3}\right) x^{2}+3 y^{2}-2 \omega_{1} x y .
$$

It is easy to check that $x=2$ is a root of $h_{1}(x, 1)$. The other root is smaller that 2 , because the product is quite close to 3 . Hence $h_{1}(x, 1) \geqslant 0$ for $x \geqslant 2$ and $h_{1}(x, y) \geqslant 0$ when $x \geqslant 2 y \geqslant 0$, which is the case with $x=J_{\tau}^{1 / 2}$ and $y=J_{v}^{1 / 2}$. Hence (5.57) yields

$$
\begin{aligned}
F^{\prime}(1) & \geqslant x^{2}+3 y^{2}-2 \omega x y \geqslant x^{2}+3 y^{2}-2 \omega_{1} x y=10^{-3} x^{2}+h_{1}(x, y) \\
& \geqslant 10^{-3} x^{2} \geqslant \frac{3}{4} \cdot 10^{-3},
\end{aligned}
$$

where we used (5.68), (5.69), and the fact that (5.66) fails.

We are now through with the cases when $\omega \leqslant \omega_{1}$, we may assume that $\omega>\omega_{1}$, and then

$$
f(2-\omega) \leqslant f\left(2-\omega_{1}\right)=f(0.251)<1.59
$$

by (5.24) and (5.25).

If $y^{2} \geqslant 1 / 5$, then (5.59) says that

$$
F^{\prime}(1) \geqslant y^{2}+3-2 f(2-\omega) \geqslant 3.2-2 f(2-\omega) \geqslant 10^{-2},
$$

and we are happy. If $x^{2} \leqslant y^{2}+\frac{8}{10}$, we use (5.58) and get that

$$
F^{\prime}(1) \geqslant-x^{2}+y^{2}+4-2 f(2-\omega) \geqslant 3.2-2 f(2-\omega) \geqslant 10^{-2},
$$

and we are happy as well. So we may assume that $y^{2}<1 / 5$ and $x^{2}>y^{2}+\frac{8}{10}$. Then $x^{2}>5 y^{2}$. We return to (5.57), consider the case when

$$
\omega_{1} \leqslant \omega \leqslant \omega_{2}=1.78,
$$


and set

$$
h_{2}(x, y)=a x^{2}+3 y^{2}-2 \omega_{2} x y,
$$

where we choose $a=\frac{2 \omega_{2} \sqrt{5}-3}{5}$. By our choice of $\omega_{2}$ (just a little smaller than $4 / \sqrt{5}$ ), $a<1-10^{-3}$.

Our choice of $a$ is such that $h_{2}(\sqrt{5}, 1)=0$. The other root of $h_{2}(x, 1)$ is smaller that $\sqrt{5}$, because the product is very close to 3 . Then $h_{2}(x, 1) \geqslant 0$ for $x \geqslant \sqrt{5}$ and $h_{2}(x, y) \geqslant 0$ for $x \geqslant \sqrt{5} y \geqslant 0$.

Note that this is the case for our usual choice of $x=J_{\tau}^{1 / 2}$ and $y=J_{v}^{1 / 2}$. Now (5.57) says that

$$
\begin{aligned}
F^{\prime}(1) & \geqslant x^{2}+3 y^{2}-2 \omega x y \geqslant x^{2}+3 y^{2}-2 \omega_{2} x y \\
& =(1-a) x^{2}+h_{2}(x, y) \geqslant(1-a) x^{2} \geqslant \frac{8}{10} \cdot 10^{-3},
\end{aligned}
$$

which is fine.

So we may now assume that $\omega>\omega_{2}$, and then,

$$
f(2-\omega) \leqslant f\left(2-\omega_{2}\right)=f(0.22)<1.53,
$$

by (5.24) and (5.25).

If $y^{2} \geqslant 8.10^{-2},(5.59)$ says that $F^{\prime}(1) \geqslant 3.08-2 f(2-\omega)>10^{-2}$, and we are happy. So we may assume that $y^{2} \leqslant 8 \cdot 10^{-2}$. We can keep our previous information that $x^{2}>y^{2}+8 / 10$, and hence $x^{2}>11 y^{2}$.

This time we set $h_{3}(x)=a x^{2}+3 y^{2}-4 x y$, with $a=\frac{4 \sqrt{11}-3}{11}<1-10^{-2}$. Note that $h_{3}(\sqrt{11}, 1)=0$ and the other root of $h_{3}(x, 1)$ is smaller. Therefore $h_{3}(x, y) \geqslant 0$ when $x \geqslant \sqrt{11} y \geqslant 0$, and in particular for our usual choice of $x$ and $y$. This time (5.57) yields

$$
F^{\prime}(1) \geqslant x^{2}+3 y^{2}-4 x y \geqslant(1-a) x^{2}+h_{3}(x, y) \geqslant(1-a) x^{2}>8 \cdot 10^{-3}
$$

because $\omega \leqslant 2$ and $x^{2} \geqslant 8 / 10$.

This proves (2.11) in our last case, and completes our proof of Proposition 2.9.

\section{Local minimizers in a ball}

Let $B$ be an open ball in the plane. For definiteness, take $B=B(0,1)$. There is a natural notion of local minimizer in $B$ that we describe now. Call a pair $(u, K)$ admissible when $K \subset B$ is closed in $B, u \in W_{\text {loc }}^{1,2}(B \backslash K)$, and $H^{1}(K \cap B(0, r))+$ $\int_{B(0, r) \backslash K}|\nabla u|^{2}<+\infty$ for all $r<1$. Competitors for $(u, K)$ are admissible pairs $(v, L)$ that satisfy (1.2), (1.3) and (1.4) for some $R<1$ (and where we replace $\mathbb{R}^{2}$ with $B$ ). Finally define local minimizers in $B$ to be admissible pairs $(u, K)$ such that, for each competitor $(v, L)$ for $(u, K)$, we have (1.6) for all $R<1$ close enough to 1 .

We do not wish to define local minimizers in $\Omega$ when $\Omega$ is a planar domain, because there may be more than one reasonable definition. However we should probably demand that if $(u, K)$ is a local minimizer in $\Omega$ and $B \subset \Omega$ is an open ball, then $(u, K)$ is a local minimizer in $B$. This way the paragraph below applies to $(u, K)$ as well. 
The main point of this section is to say officially that if $(u, K)$ is a reduced local minimizer in $B(0,1)$, then all the propositions of Section 2 stay true when we restrict to radii $r<1$, and with the same proof. The same comment stays true for most of the results of the next sections (those that make sense locally). We shall not insist.

A more delicate question concerns minimizers of the (usual) Mumford-Shah functional in planar domains associated with an $L^{\infty}$ function $g$. (See Section 13 for a definition.) It is not clear at all that some version of Proposition 3.5 holds in the general case but we can always get results indirectly, using blow-up arguments as in [4].

However, with the extra assumption that $g$ is a Lipschitz function on the domain, Proposition 3.5 stays true up to an additive $\mathrm{O}(r)$ term. In the proofs of the various monotonicity inequalities we lose an $\mathrm{O}(r)$ each time we evaluate $\frac{E(r)+\ell(r)}{r}$ because of the extra term $\int(u-g)^{2} d x$ which is an $\mathrm{O}\left(r^{2}\right)$. This shows that Propositions 2.7, 2.9, 2.12 and 2.13 hold with an extra $\mathrm{O}(r)$ term. Once this is known many other results in the sequel (like say Theorem 9.1) hold true for minimizers of the usual MumfordShah functional associated with a Lipschitz function $g$ under a sufficiently small scale depending on the $L^{\infty}$ and Lipschitz norm of $g$.

\section{7. $C^{1}$ curves and spiders}

The following definitions, although somewhat heavy, will be convenient. Let $(u, K) \in$ $R G M$, as usual, and $B=B(x, r)$ be a disk centered on $K$. We shall say that $K \cap \bar{B}$ is a nice $C^{1}$ curve if $\Gamma=K \cap \bar{B}$ is a simple $C^{1}$ curve with its two endpoints on $\partial B$. We also require that $\Gamma$ be the image, under a rotation of $\mathbb{R}^{2}$, of the graph of some $\frac{1}{10}$-Lipschitz function $: \mathbb{R} \rightarrow \mathbb{R}$. This last condition is convenient because it gives some uniformity, and also $K \cap \bar{B}(x, \rho)$ is a nice $C^{1}$ curve for $\rho<r$ because of it.

By $C^{1}$ spider we shall mean a set composed of three simple $C^{1}$ curves that all start from a same origin (the center of the spider), make $120^{\circ}$ angles with each other at this point, and are otherwise disjoint.

We shall say that $K \cap \bar{B}(x, r)$ is a nice spider if $K \cap \bar{B}(x, r)$ is a $C^{1}$ spider with center in $B\left(x, \frac{r}{2}\right)$, the extremities of the three curves that compose it lie in $\partial B(x, r)$, and also each of these curves is the image under a rotation of a $\frac{1}{10}$-Lipschitz graph. The center of such a spider will be called a spider point of $K$.

Finally we say that $B(x, r)$ is a good curve or spider disk when $x \in K$ and either $K \cap \bar{B}(x, r)$ is a nice $C^{1}$ curve, or else $K \cap \bar{B}(x, 4 r)$ is a nice spider. The point of this weird definition is that it accomodates nicely the case when $K \cap \bar{B}(x, 4 r)$ is a spider with its centre close to $\partial B(x, r)$, so that $B(x, \rho)$ stays a good curve or spider disk for $\rho<r$. But of course there is nothing too important about these definitions.

We shall need the following result to provide us with good curve or spider disks.

THEOREM 7.1. - There exist constants $\varepsilon_{0}>0$ and $a_{0}>0$ such that if $(u, K) \in$ $R G M, x \in K$, and $r>0$ are such that

$$
\int_{B(x, r) \backslash K}|\nabla u|^{2} \leqslant \varepsilon_{0} r,
$$

then $B\left(x, a_{0} r\right)$ is a good curve or spider disk. 
This is Proposition 35 on p. 445 of [15]. It is not too hard to deduce from the main results of [7] via a compactness argument.

We shall need later the following simple result.

PROPOSITION 7.3. - For each $\varepsilon>0$ we can find a constant $a=a(\varepsilon)>0$ such that if $(u, K) \in R G M$, and $B(x, r)$ is a nice curve or spider disk, then

$$
\int_{B(x, a r) \backslash K}|\nabla u|^{2} \leqslant \varepsilon a r,
$$

and there is a propeller $P$ through $x$ (but whose center may be out of $B(x, r)$ ) such that

$$
\operatorname{dist}(y, P) \leqslant \text { ear for all } y \in K \cap B(x, \text { ar }) .
$$

This is far from new, and there are many ways to get it. The simplest here is probably to prove first that

$$
\int_{B(x, t r) \backslash K}|\nabla u|^{2} \leqslant 2 \pi t^{4 / 3} r \quad \text { for } t \leqslant 1 .
$$

To prove (7.6) notice that by assumption all the points of $K \cap \partial B(x, t r)$ lie in the same connected component of $K$ when $t<1$. Then we can apply the argument of Bonnet described in Lemma 5.48 and get the analogue of (5.56) here. That is,

$$
E(t r)=\int_{B(x, t r) \backslash K}|\nabla u|^{2} \leqslant \frac{\lambda}{2} \omega^{2} t r J_{\tau}+\frac{t r}{2 \lambda} J_{\nu},
$$

where $J_{\tau}=\int_{\partial B(x, t r) \backslash K}\left(\frac{\partial u}{\partial \tau}\right)^{2}, J_{v}=\int_{\partial B(x, t r) \backslash K}\left(\frac{\partial u}{\partial \nu}\right)^{2}$, and $\pi \omega t r$ is the length of the longest component of $\partial B(x, t r) \backslash K$. Since $B(x, r)$ is a nice curve or spider disk, we easily get that $\omega \leqslant 3 / 2$, say. Then we take $\lambda=2 / 3$ in (7.7) and get that

$$
E(t r) \leqslant \frac{3}{4} t r \int_{B(x, t r) \backslash K}|\nabla u|^{2}=\frac{3}{4} \operatorname{tr} E^{\prime}(t r)
$$

for $t \leqslant 1$. Then we integrate, use (2.4) for $t=1$, and get (7.6).

Of course (7.4) is trivial to get with (7.6). The argument for (7.5) is very standard (see for instance [7] or [4]), so we only sketch it. Because of (7.6) we can find $\rho \in(\mathrm{ar}, 2 \mathrm{ar})$ such that $\int_{\partial B(x, \rho) \backslash K}|\nabla u|^{2} \leqslant c a^{1 / 3}$. Then we compare $(u, K)$ with a competitor $(v, L)$ where $L \cap B(x, \rho)$ is a piece of line or propeller that connects the 2 or 3 points of $K \cap \partial B(x, \rho)$ and $v$ is obtained using Corollary 4.15, for instance. The reader may be worried about the case when $K \cap B(x, r)$ is a spider and its center is very near $\partial B(x, \rho)$, but this case is easily avoided by choosing $\rho$ more carefully. The computations give that $H^{1}(K \cap B(x, \rho))$ is almost optimal, and (7.5) follows. 


\section{Limits of sequences of minimizers}

We want to say that sequences in RGM have subsequences that converge, and that the limits lie in RGM. But let us first define convergence. Let $\left\{K_{n}\right\}$ be a sequence of closed sets in the plane. We say that $\left\{K_{n}\right\}$ converges to $K$ if $K \subset \mathbb{R}^{2}$ is closed and

$$
\lim _{n \rightarrow+\infty} d_{R}\left(K_{n}, K\right)=0
$$

for each $R>0$, where

$$
\begin{aligned}
d_{R}\left(K_{n}, K\right)= & \sup \left\{\operatorname{dist}(x, K) ; x \in K_{n} \cap B(0, R)\right\} \\
& +\sup \left\{\operatorname{dist}\left(x, K_{n}\right) ; x \in K \cap B(0, R)\right\}
\end{aligned}
$$

is a lot like the Hausdorff distance in $B(0, R)$. We use the convention that if $K_{n} \cap B(0, r)$ is empty, then the first sup is zero (and similary for the second one). Thus $\left\{K_{n}\right\}$ can converge to $\emptyset$ if the sets $K_{n}$ go away to $\infty$.

Note also that $d_{R}$ is a nondecreasing function of $R$, so we may restrict to a sequence of radii $R$ that tends to $+\infty$. Because of this and the classical properties of Hausdorff distance, we can extract from any given sequence $\left\{K_{n}\right\}$ a subsequence that converges.

Next consider a sequence $\left\{\left(u_{n}, K_{n}\right)\right\}$ of admissible pairs (see Section 1$)$, and let $(u, K)$ be an admissible pair. We say that $\left\{\left(u_{n}, K_{n}\right)\right\}$ converges to $(u, K)$ if $\left\{K_{n}\right\}$ converges to $K$ as above, and in addition $\left\{\nabla u_{n}\right\}$ converges to $\{\nabla u\}$ uniformly on $H$ for every compact subset $H$ of $\mathbb{R}^{2} \backslash K$.

Note that this makes sense because if $H \subset \mathbb{R}^{2} \backslash K$ is compact, then it is contained in $\mathbb{R}^{2} \backslash K_{n}$ for $n$ large. The reader may fear that requiring uniform convergence is too strong (because we only know that $\nabla u_{n} \in L_{\text {loc }}^{2}$ ), but this will not matter because we shall only consider global minimizers for which we have much better estimates.

LEMMA 8.3. - If $\left(u_{n}, K_{n}\right) \in R G M$ for $n \geqslant 0$, then we can extract a subsequence $\left\{n_{k}\right\}$ for which $\left\{\left(u_{n_{k}}, K_{n_{k}}\right)\right\}_{k \geqslant 0}$ converges.

This is standard. We can extract a first subsequence to make the sets $K_{n}$ converge. Then we notice that $u_{n}$ is harmonic on $\mathbb{R}^{2} \backslash K_{n}$, and that we have the uniform bounds

$$
\int_{B(0, R) \backslash K_{n}}\left|\nabla u_{n}\right|^{2} \leqslant 2 \pi R
$$

for each $R>0$ (as in (2.4)). Thus if $H \subset \mathbb{R}^{2} \backslash K$ is compact we have uniform bounds on $\left\|\nabla^{2} u_{n}\right\|_{L^{\infty}(H)}$ for $n$ large, and the lemma follows by Montel.

THEOREM 8.5. - If $\left\{\left(u_{n}, K_{n}\right)\right\}$ is a sequence in RGM that converges to some limit $(u, K)$, then $(u, K) \in R G M$.

This is Theorem 31 on p. 444 of [15], but the proof is almost exactly the same as in [4], where blow-up sequences of (usual) reduced Mumford-Shah minimizers are shown to converge to reduced global minimizers (modulo extracting subsequences). Of course we may apply Theorem 8.5 to sequences obtained from a single minimizer $\left(u_{0}, K_{0}\right)$ by various translations, dilations, etc. 


\section{Miscellaneous properties of $\mathbf{K}$}

We continue with our assumption that $(u, K) \in R G M$ and use the monotonicity propositions of Section 2 to prove various results on $K$. We start with results relative to the existence of long connected sets in $K$.

THEOREM 9.1. - There is an absolute constant $a_{1}>0$ such that $x \in K, r>0$, and $K$ contains two disjoint connected sets that both meet $\partial B\left(x, a_{1} r\right)$ and $\partial B(x, r)$, then $B\left(x, a_{1} r\right)$ is a nice curve or spider disk.

See the beginning of Section 7 for the meaning of the conclusion. The proof of this theorem will keep us busy for a good part of this section, but let us first state and prove a consequence.

COROLLARY 9.2. - If $K$ contains two disjoint unbounded connected sets, then $K$ is a line or a propeller and $u$ is locally constant on $\mathbb{R}^{2} \backslash K$ (as in our examples (1.9), (1.10)).

Indeed, we may as well assume that the origin lies on $K$. Then Theorem 9.1 tells us that $B\left(0, a_{1} r\right)$ is a nice curve or spider disk for $r$ large enough. In particular, $K \cap B\left(0, a_{1} r\right)$ or $K \cap B\left(0,4 a_{1} r\right)$ is connected. This proves that $K$ itself is connected, and the result follows from [4]. We can also use Proposition 7.3 instead of [4] to conclude. For each choice of $R>0$ and $\varepsilon>0$ (small) we apply the proposition to the disk $B(0, r)$, where $r=a(\varepsilon)^{-1} R$, and get that $K \cap B(0, r)$ is $\varepsilon R$-close to a line or a propeller. The conclusion follows.

To prove the theorem we may assume that $x=0$ and $r=1$. Call $\gamma_{1}, \gamma_{2}$ the two connected subsets of $K$ that are given by assumption. We shall first prove the conclusion in the special case when

$\gamma_{1}, \gamma_{2}$ are contained in the same connected component of $K$.

Note that for $a_{1} \leqslant \rho \leqslant 1, \gamma_{1}$ and $\gamma_{2}$ meet $\partial B(0, \rho)$. This means that if $N(\rho)$ is the number of points in $K \cap \partial B(0, \rho)$, then $N(\rho) \geqslant 2$. Moreover, if $N(\rho)=2$, the two points of $K \cap \partial B(0, \rho)$ lie on $\gamma_{1} \cup \gamma_{2}$, hence on the same component of $K$ (by (9.3)). If in addition $\rho \in \mathcal{R}$ (the set of full measure that shows up in the propositions of Section 2), then Proposition 2.9 applies and

$$
\rho F^{\prime}(\rho) \geqslant 10^{-10} \int_{\partial B(0, \rho) \backslash K}|\nabla u|^{2} .
$$

Now (2.4), the definitions (2.1)-(2.3), and Propositon 2.5 say that

$$
\begin{aligned}
4 \pi & \geqslant F(1) \geqslant F(1)-F\left(a_{1}\right) \geqslant \int_{\mathcal{R} \cap\left(a_{1}, 1\right)} F^{\prime}(\rho) d \rho \\
& \geqslant 10^{-10} \int_{a_{1}}^{1}\left\{\int_{\partial B(0, \rho) \backslash K}|\nabla u|^{2} d H^{1}\right\} \frac{d \rho}{\rho} .
\end{aligned}
$$

Let $a_{0}$ and $\varepsilon_{0}$ denote the two constants of Theorem 7.1. Choose $\lambda>0$ smaller than $a_{0}$ and such that $2 \pi \lambda<\varepsilon_{0} / 2$. Then choose an integer $N$ so large that $4 \pi \cdot 10^{10} / N<\varepsilon_{0} / 2$. 
Then take $a_{1}=\lambda^{N}$, and decompose the interval $\left(a_{1}, 1\right)$ into the $I_{k}=\left(\lambda^{k+1}, \lambda^{k}\right), 0 \leqslant k \leqslant$ $N-1$. Because of (9.5) we can find $k$ such that

$$
\int_{I_{k}}\left\{\int_{\partial B(0, \rho) \backslash K}|\nabla u|^{2}\right\} \frac{d \rho}{\rho} \leqslant \frac{4 \pi \cdot 10^{10}}{N} \leqslant \frac{\varepsilon_{0}}{2} .
$$

Then

$$
\begin{aligned}
\int_{B\left(0, \lambda^{k}\right) \backslash K}|\nabla u|^{2} & =\int_{B\left(0, \lambda^{k+1}\right) \backslash K}|\nabla u|^{2}+\iint_{I_{k}}\left\{\int_{\partial B(0, \rho) \backslash K}|\nabla u|^{2}\right\} d \rho \\
& \leqslant 2 \pi \lambda^{k+1}+\lambda^{k} \int_{I_{k}}\left\{\int_{\partial B(0, \rho) \backslash K}|\nabla u|^{2}\right\} \frac{d \rho}{\rho} \\
& \leqslant 2 \pi \lambda^{k+1}+\frac{\varepsilon_{0}}{2} \lambda^{k} \leqslant \varepsilon_{0} \lambda^{k}
\end{aligned}
$$

by (2.4), (9.6), and the definition of $\lambda$.

We may now apply Theorem 7.1 and get that $B\left(0, a_{0} \lambda^{k}\right)$ is a good curve or spider disk. And so is $B\left(0, a_{1}\right)$ because $a_{1} \leqslant a_{0} \lambda^{k}$. (See the comments before Theorem 7.1.)

This completes our verification of Theorem 9.1 when (9.3) holds.

Remark 9.8. - Our proof shows that $B\left(x, a_{1} r\right)$ is a nice curve or spider disk as soon as (9.4) holds for all $\rho \in \mathcal{R} \cap\left(a_{1}, 1\right)$, regardless of any special assumption on $K$.

For the general case of Theorem 9.1 we want to proceed by contradiction and compactness. So let us assume that for each integer $n \geqslant 0$ we can find $\left(u_{n}, K_{n}\right) \in R G M$ such that $0 \in K_{n}, K_{n}$ contains two disjoint connected sets $\gamma_{1, n}$ and $\gamma_{2, n}$ that both meet $\partial B\left(0,2^{-n}\right)$ and $\partial B(0,1)$, but yet for which $B\left(0,2^{-n}\right)$ is not a good curve or spider disk.

First we want to replace $\gamma_{i, n}, i=1,2$, with a simple curve $\gamma_{i, n}^{\prime} \subset \gamma_{i, n}$ that is contained in $\bar{B}(0,1) \backslash B\left(0,2^{-n}\right)$ and still connects $\partial B\left(0,2^{-n}\right)$ to $\partial B(0,1)$. Choose points $x_{i} \in \gamma_{i, n} \cap \partial B\left(0,2^{-n}\right)$ and $y_{i} \in \gamma_{i, n} \cap \partial B(0,1)$ and note that we can find rectifiable curves $\gamma_{i}$ supported on $\gamma_{i, n}$ that go from $x_{i}$ to $y_{i}$. This is because $\gamma_{i, n}$ is connected and $H^{1}\left(\gamma_{i, n} \cap B(0, R)\right)<+\infty$ for every $R>0$; see for instance [12], or Lemma 19.2 in [5]. Then we can get $\gamma_{i, n}^{\prime}$ from $\gamma_{i}$ either directly by removing the unnecessary loops (as in [12]), or by choosing $\gamma_{i}$ with the shortest length (as in Lemma 19.14 of [5], following a suggestion of Morel and Solimini).

Since we may as well replace $\left\{\left(u_{n}, K_{n}\right)\right\}$ by a subsequence, Lemma 8.3 and Theorem 8.5 tell us that we may assume that $\left\{\left(u_{n}, K_{n}\right)\right\}$ converges to some limit $(u, K) \in R G M$. We may also assume that each $\left\{\gamma_{i, n}^{\prime}\right\}$ converges to a curve $\left\{\gamma_{i}\right\}$. More precisely, we know that all the $\gamma_{i, n}^{\prime}$ have lengths at most $2 \pi$ (by (2.4)), hence we can find $2 \pi$-Lipschitz parameterizations of them defined on the limit interval, and it is easy to extract subsequences for which the parameterizations converge uniformly.

The curves $\gamma_{1}$ and $\gamma_{2}$ are contained in $\bar{B}(0,1) \cap K$ and go from 0 to $\partial B(0,1)$, but we would like to see to which extent they are disjoint. Let us check that

$$
\gamma_{1} \cap \gamma_{2} \cap B(0,1)=\{0\} .
$$


Let $x \in \gamma_{1} \cap B(0,1) \backslash\{0\}$ be given. Set $r=\frac{1}{2} \min (|x|, 1-|x|)>0$. Choose $x_{n} \in \gamma_{1, n}^{\prime}$ such that $\lim _{n \rightarrow \infty} x_{n}=x$. Recall that $\gamma_{1, n}^{\prime}$ is a simple curve from $\partial B\left(0,2^{-n}\right)$ to $\partial B(0,1)$. Thus for $n$ large enough $\gamma_{1, n}^{\prime} \backslash\left\{x_{n}\right\}$ has two connected components, which we call $\gamma_{1, n}^{+}$ and $\gamma_{1, n}^{-}$. Moreover, if $\left|x_{n}-x\right|<r, \gamma_{1, n}^{ \pm}$meets $\partial B\left(x_{n}, \rho\right)$ for all $\rho \in(0, r)$. Since $\gamma_{1, n}^{+}$, $\gamma_{1, n}^{-}$obviously lie on the same component of $K_{n}$, we can apply Theorem 9.1 (when (9.3) holds) and get that $B\left(x_{n}, a_{1} r\right)$ is a nice curve or spider disk for $K_{n}$.

If $K_{n} \cap B\left(x_{n}, a_{1} r\right)$ is a nice $C^{1}$ curve, it is obviously contained in $\gamma_{1, n}^{\prime}$ (which is simple), and $\gamma_{2, n}^{\prime}$ does not meet $B\left(x_{n}, a_{1} r\right)$ (because it is disjoint from $\gamma_{1, n}^{\prime}$ ). Even in the case when $K_{n} \cap B\left(x_{n}, 4 a_{1} r\right)$ is a nice spider, we can check that $\gamma_{2, n}$ does not meet $B\left(x_{n}, 4 a_{1} r\right)$, because there is not enough room in $K_{n} \cap B\left(x_{n}, 4 a_{1} r\right)$ for two disjoint simple curves that cross $B\left(x_{n}, 4 a_{1} r\right)$. So $\operatorname{dist}\left(x_{n}, \gamma_{2, n}^{\prime}\right) \geqslant a_{1} r$ in all cases. We may now go to the limit and get that $\operatorname{dist}\left(x, \gamma_{2}\right) \geqslant a_{1} r$, from which (9.9) follows.

Each $\gamma_{i}$ contains a simple curve $\gamma_{i}^{\prime}$ that goes from 0 to $\partial B(0,1)$. Here it is not too important that the curves be simple, but we want them to leave 0 immediately and never come back. This allows us to apply the special case of Theorem 9.1 where (9.3) holds to the disk $B(0,1)$ and the disjoint connected sets $\gamma_{i}^{\prime} \backslash\{0\} \subset K$. We get that $B\left(0, a_{1}\right)$ is a nice curve or spider disk for $K$.

Now let be $\varepsilon_{0}$ as in Theorem 7.1, and apply Proposition 7.3 with $\varepsilon=\varepsilon_{0} / 2$. This gives that

$$
\int_{B\left(0, a a_{1}\right) \backslash K}|\nabla u|^{2} \leqslant \frac{\varepsilon_{0} a a_{1}}{2},
$$

with $a=a\left(\varepsilon_{0} / 2\right)$. Next we want to use the fact that

$$
\int_{B\left(0, a a_{1}\right) \backslash K}|\nabla u|^{2}=\lim _{n \rightarrow+\infty} \int_{B\left(0, a a_{1}\right) \backslash K_{n}}\left|\nabla u_{n}\right|^{2} .
$$

Note that in (9.11) we shall need the inequality that does not come from Fatou's lemma, and which is not trivial. It is a byproduct of the proof that $(u, K)$ is a global minimizer; see Theorem 2.2 in [4]. Now

$$
\int_{B\left(0, a a_{1}\right) \backslash K_{n}}\left|\nabla u_{n}\right|^{2}<\varepsilon_{0} a a_{1}
$$

for $n$ large enough, by (9.11) and (9.10), Theorem 7.1 says that $B\left(0, a_{0} a a_{1}\right)$ is a good curve or spider disk for $K_{n}$, and this contradicts our assumption that $B\left(0,2^{-n}\right)$ is not a good curve or spider disk.

Note that we did not really need to use (9.11) here, because it was enough to prove that

$$
\int_{B\left(0, a a_{1}\right) \backslash K_{n}}\left|\nabla u_{n}\right| \leqslant \varepsilon\left(a a_{1}\right)^{3 / 2}
$$

for a small enough $\varepsilon$. The point is that we also know from our earlier application of Proposition 7.3 that all points of $K \cap B\left(0, a a_{1}\right)$ are very close to some propeller $P$, hence the same thing holds for $K_{n} \cap B\left(0, \frac{1}{2} a a_{1}\right)$ for $n$ large. Then we can apply 
Theorem 4.6 p. 802 of [7], or its analogue for spiders (essentially proved in Section 10 of [7], but unfortunately with no explicit statement to refer to), and get the desired contradiction. The estimate (9.13) is easier to obtain than (9.11); it follows from the uniform convergence of $\left\{\nabla u_{n}\right\}$ to $\nabla u$ on compact subsets of $\mathbb{R}^{2} \backslash K$, the uniform $L^{2}$ estimates (8.4), Hölder, and the fact that $K$ has neighborhoods with arbitrarily small Lebesgue measure. We skip the details.

This completes our proof of Theorem 9.1. Our next result is a (slightly simpler) variant of Theorem 9.1.

PROPOSITION 9.14. - There is an absolute constant $a_{2}>0$ such that if $x \in K, r>0$, and if we can find two distinct connected components of $B(x, r) \backslash K$ that meet $\partial B(x, \rho)$ for $a_{2} r<\rho<r$, then $B\left(x, a_{2} r\right)$ is a nice curve or spider disk.

As before it is enough to prove the proposition when $x=0$ and $r=1$. We want to verify that (9.4) holds for all radii $\rho \in \mathcal{R} \cap\left(a_{2}, 1\right)$. If we succeed, the proposition will follow from Remark 9.8.

So let $\rho \in \mathcal{R} \cap\left(a_{2}, 1\right)$ be given. If $N(\rho) \geqslant 3$, (9.4) follows from Proposition 2.9. The only other option is that $N(\rho)=2$, because our two distinct components of $B(0,1) \backslash K$ (call them $\Omega_{1}$ and $\Omega_{2}$ ) meet $\partial B(0, \rho)$. Let $a$ and $b$ denote the points of $K \cap \partial B(0, \rho)$. We want to show that

$$
a \text { and } b \text { lie in the same component of } K \text {, }
$$

because then Proposition 2.9 will tell us that (9.4) holds, as needed.

Call $I_{1}, I_{2}$ the two components of $\partial B(0,1) \backslash K$. Recall that our components $\Omega_{1}, \Omega_{2}$ both meet $\partial B(0, \rho)$. Hence $I_{1}$ and $I_{2}$ lie in different components of $B(0,1) \backslash K$ (the two $\left.\Omega_{i}\right)$. In particular $K \cap \bar{B}(0, \rho)$ separates $I_{1}$ from $I_{2}$ in $\bar{B}(0, \rho)$. By a standard fact of two-dimensional topology (see Theorem 14.3 on p. 123 of [20]), there is a connected component of $K \cap \bar{B}(0, \rho)$ that still separates $I_{1}$ from $I_{2}$ in $\bar{B}(0, \rho)$. Obviously this component contains $a$ and $b ;(9.4)$ and Proposition 9.14 follow.

COROLLARY 9.16. - If $\mathbb{R}^{2} \backslash K$ is not connected, then $K$ is a line or a propeller, and $u$ is locally constant on $\mathbb{R}^{2} \backslash K$.

For this we need to know that

$\mathbb{R}^{2} \backslash K$ never has any bounded connected component.

This is standard. The first proof is in [4], but one can also look in Section 15 of [5]. It is also fairly easy. The idea is to find a piece of $C^{1}$ curve in the boundary of our bounded component $\Omega$, and then open a hole in it. This is authorized by (1.4) because $\Omega$ is bounded, and if the hole is small enough we can modify the values of $u$ in $\Omega$ to get a function which is smooth across the hole, without paying more than half the size of the hole in energy.

The corollary is now easy to prove. We may assume that $0 \in K$. By assumption, we have two distinct components in $\mathbb{R}^{2} \backslash K$. These components are unbounded by (9.17), and so they meet $\partial B(0, \rho)$ for all $\rho$ large enough. Proposition 9.14 says that $B(0, r)$ is 
a nice curve or spider disk for $r$ large enough, and we can conclude as in Corollary 9.2 (for instance, $K$ is connected).

PROPOSITION 9.18. - If $K$ is symmetric with respect to the origin, then $K=\emptyset$ or $K$ is a line, and $u$ is locally constant on $\mathbb{R}^{2} \backslash K$.

Let $\rho \in \mathcal{R}$ be given. If $N(\rho)=0$, then (9.4) holds because of Proposition 2.7. If $N(\rho)>0$, then $N(\rho) \geqslant 2$ and all components of $\partial B(0, \rho) \backslash K$ have lengths at most $\pi \rho$, by symmetry. Hence (9.4) holds because of Proposition 2.13 .

So (9.4) holds for all $\rho \in \mathcal{R}$, and we can continue as in the proof of Theorem 9.1 when (9.3) holds. We get radii $r$ as large as we want such that

$$
\int_{B(0, r) \backslash K}|\nabla u|^{2} \leqslant \varepsilon_{0} r,
$$

as in (9.7). If $0 \in K$, we can apply Theorem 7.1 just as before and conclude. The other case in not much harder; if $K$ is not empty, we can choose a fixed origin $y \in K$ and note that $\int_{B(y, r-|y|) \backslash K}|\nabla u|^{2} \leqslant \varepsilon_{0} r$, which is essentially as good as (9.19) (especially for $r$ large). Our argument says that $B(y, \rho)$ is a good curve or spider disk for arbitrarily large values of $\rho$, which is enough to conclude.

\section{Description of a connected component of $K$}

We still assume here that $(u, K) \in R G M$, as in Section 1 . Let $K_{0}$ be a connected component of $K$, and assume that it is not reduced to one point. We want to give a reasonably precise description of $K_{0}$. We start by recalling a result from [4].

PROPOSITION 10.1. - The set $K_{0}$ is a chord-arc tree. By this we mean that if $x, y$ are two distinct points of $K_{0}$, there is a unique simple arc $\gamma_{x, y} \subset K_{0}$ that connects $x$ to $y$, and

$$
\text { length }\left(\gamma_{x, y}\right) \leqslant C_{1}|x-y|
$$

Here $C_{1}$ is an absolute constant.

Let us rapidly describe the argument. First observe that

$$
K \text { contains no loop }
$$

(i.e., no simple closed curve). This follows from (9.17). Let $x, y \in K_{0}$ be given, with $x \neq y$. Because $K_{0}$ is connected and $H^{1}\left(K_{0} \cap B(0, r)\right)<+\infty$ for every $R>0$, we can find a rectifiable curve $\gamma_{x, y}^{\prime} \subset K_{0}$ from $x$ to $y$. See Lemma 19.2 in [5], for instance, but the argument is standard. From this it is easy to deduce the existence of a simple arc $\gamma_{x, y} \subset K_{0}$ that goes from $x$ to $y$. One can start from $\gamma_{x, y}^{\prime}$ and remove the loops, as in [12], or take $\gamma_{x, y}^{\prime}$ directly with minimal length and show that it is simple, as in Lemma 19.14 of [5].

Next the arc $\gamma_{x, y}$ is unique, because of (10.3). The argument is simple, and even written in [5], just after (31.5). So we are just left with (10.2) to prove. Let us check the following apparently more general (but in fact equivalent) result. 
LEMmA 10.4. - If $\gamma \subset K$ is a simple curve, then it is chord-arc. This means that if $x, y \in \gamma$, the length of the arc of $\gamma$ between $x$ and $y$ is at most $C_{1}|x-y|$.

This lemma is the only place where our proof will differ from the argument in [4]. Let $\gamma, x$, and $y$ be as in its statement, and call $\gamma^{\prime}$ the arc of $\gamma$ between $x$ and $y$. Let us already check that

$$
\gamma^{\prime} \subset B\left(x, 3|x-y| / a_{1}\right),
$$

where $a_{1}$ is the constant in Theorem 9.1, which we can safely assume to be smaller than $1 / 10$.

Assume not, and choose $z \in \gamma^{\prime} \backslash B\left(x, 3|x-y| / a_{1}\right)$. Call $\gamma_{1}$ and $\gamma_{2}$ the two connected components of $\gamma^{\prime} \backslash\{z\}$. Also set $r=2|x-y| / a_{1}$. Both curves $\gamma_{i}$ meet $B\left(x, a_{1} r\right)$ (because they contain $x$ or $y$ ) and $\partial B(x, r)$ (because of $z$ ). Hence we can apply Theorem 9.1 (and even the special case when (9.3) holds). We get that $B(x, 2|x-y|)$ is a nice curve or spider disk. But then there is a simple curve from $x$ to $y$ which is contained in $K \cap B(x, 8|x-y|)$. This curve does not contain $z$, hence its existence contradicts the uniqueness in Proposition 10.1, and (10.5) follows. Now

$$
\text { length }\left(\gamma^{\prime}\right)=H^{1}\left(\gamma^{\prime}\right) \leqslant H^{1}\left(K \cap B\left(x, 3|x-y| / a_{1}\right)\right) \leqslant \frac{6 \pi}{a_{1}}|x-y|
$$

because $\gamma^{\prime}$ is simple, and by (10.5) and (2.4). This completes our proof of Lemma 10.4 and Proposition 10.1.

Next we want to show that our connected component $K_{0}$ of $K$ is a piecewise $C^{1}$ (but with possibly infinitely many pieces) tree, with some estimates. Let us introduce a few ways to measure how far from the extremities of $K_{0}$ is a given point $x \in K_{0}$. First set

$$
r_{1}(x)=\sup \{r>0 ; B(x, r) \text { is a good curve or spider disk }\} .
$$

(See the beginning of Section 7 for a definition.) When there is no $r>0$ as in (10.7), set $r_{1}(x)=0$. The next quantity $r_{2}(x)$ measures how far we can run in $K$ away from $x$ in at least two different directions. Set

$$
\begin{array}{r}
r_{2}(x)=\sup \{r \geqslant 0 \text {; there is an injective Lipschitz function } z:[-r, r] \rightarrow K \\
\text { such that } \left.z(0)=x \text { and }\left|z^{\prime}(t)\right|=1 \text { almost-everywhere }\right\} .
\end{array}
$$

A third quantity will be the distance to the set $K^{\sharp}$ of points of high energy. That is,

$$
K^{\sharp}=\left\{x \in \mathbb{R}^{2} ; \limsup _{r \rightarrow 0} \frac{1}{r} \int_{B(x, r) \backslash K}|\nabla u|^{2}>0\right\} .
$$

This is the original definition from [15], but recall that

$$
K^{\sharp}=\left\{x \in K ; r_{1}(x)=0\right\} .
$$

Indeed, $K^{\sharp} \subset K$ because $u$ is harmonic (hence smooth) on $\mathbb{R}^{2} \backslash K$. If $x \in K$ and $r_{1}(x)>0$, Proposition 7.3 tells us that $x \notin K^{\sharp}$. Conversely, if $x \in K \backslash K^{\sharp}$ (or even if $\inf _{r}\left\{\frac{1}{r} \int_{B(x, r) \backslash K}|\nabla u|^{2}\right\}<\varepsilon_{0}$ ), Theorem 7.1 says that $r_{1}(x)>0$. This proves (10.10). 
A minor variant of the distance to $K^{\sharp}$ is the distance to

$$
\operatorname{tip}\left(K_{0}\right)=K_{0} \cap K^{\sharp}=\left\{x \in K_{0} ; r_{1}(x)=0\right\} .
$$

(We shall also see that $\operatorname{tip}\left(K_{0}\right)=\left\{x \in K_{0} ; r_{2}(x)=0\right\}$.)

We shall need later a last radius $r_{3}(x)$, which roughly measures how far away the pathes in $\mathbb{R}^{2} \backslash K$ that connect the different components of $B(x, \rho) \backslash K, \rho$ small, have to go. The simplest is to define $r_{3}(x)$ first when $x$ is a regular point of $K$, i.e., when we can find $\rho>0$ such that $K \cap B(x, \rho)$ is a nice $C^{1}$ curve. Then fix any such $\rho>0$ and set

$$
\begin{array}{r}
r_{3}(x)=\inf \{r>0 ; \text { there is a curve } \gamma \subset B(x, r) \backslash K \text { that connects } \\
\text { the two components of } B(x, \rho) \backslash K\} .
\end{array}
$$

If there is no such $r>0$, set $r_{3}(x)=+\infty$. This is not an important case, because Corollary 9.16 tells us that then $K$ is a line or propeller. Also note that $r_{3}(x)$ does not depend on our choice of $\rho>0$.

When $x \in K^{\sharp}$, set $r_{3}(x)=0$. We shall see later that this is natural, because $r_{3}(y)$ tends to 0 when $y$ is a regular point of $K$ that tends to $x$. When there is a $\rho>0$ such that $K \cap B(x, \rho)$ is a spider centered at $x$, choose any number $r_{3}(x)$ such that

$$
\liminf _{y \rightarrow x} r_{3}(y) \leqslant r_{3}(x) \leqslant \limsup _{y \rightarrow x} r_{3}(y) .
$$

We are now ready to state a proposition that says that all these numbers are equivalent.

PROPOSITION 10.14. - There is an absolute constant $C>1$ such that

$$
r_{1}(x) \leqslant \operatorname{dist}\left(x, K^{\sharp}\right) \leqslant \operatorname{dist}\left(x, \operatorname{tip}\left(K_{0}\right)\right) \leqslant r_{3}(x) \leqslant C r_{2}(x) \leqslant C^{2} r_{1}(x)
$$

for $x \in K_{0}$.

Let us start our proof with the most interesting case when $r_{2}(x)>0$. Pick $r<r_{2}(x)$ close to $r_{2}(x)$. By the definition (10.8), we can find a Lipschitz injective function $z:[-r, r] \rightarrow K$ such that $z(0)=x$ and $\left|z^{\prime}(t)\right|=1$ almost-everywhere. Set $\gamma_{+}=z([0, r])$ and $\gamma_{-}=z([-r, 0])$. Note that length $\left(\gamma_{ \pm}\right)=r$, and so Lemma 10.4 tells us that $|z( \pm r)-x| \geqslant \frac{r}{C_{1}}$. In particular, the disjoint arcs $\gamma_{ \pm}$both meet $\partial B(x, \rho)$ for $0<\rho \leqslant \frac{r}{C_{1}}$. By Theorem 9.1, $B\left(x, \frac{a_{1} r}{C_{1}}\right)$ is a good curve or spider disk, and $r_{1}(x) \geqslant \frac{a_{1} r}{C_{1}}$. Since we could choose any $r<r_{2}(x)$, we get that

$$
r_{1}(x) \geqslant \frac{a_{1} r_{2}(x)}{C_{1}} .
$$

To continue our argument we need a maximal extension of $z$. We can find an interval $I \subset \mathbb{R}$ that contains $[-r, r]$ and an injective Lipschitz extension of $z$ to $I$ such that $z(I) \subset K,\left|z^{\prime}(t)\right|=1$ almost-everywhere on $I$, and such that there is no further extension of $z$ with the same properties and to an interval that contains $I$ strictly. Such a maximal extension exists by standard arguments that we do not wish to repeat here.

Suppose that $I \neq \mathbb{R}$, and let $a$ be any extremity of $I$. Then we can define $z(a)$, even if $a \notin I$, because $z$ is Lipschitz. Let us check that $z(a) \in \operatorname{tip}\left(K_{0}\right)$. If not, (10.11) tells us 
that $r_{1}(z(a))>0$ and $B(z(a), \rho)$ is a good curve or spider disk for $\rho$ small. Then it is possible to extend $z$ to some little neighborhood $V$ of $a$, in such a way that $z$ is injective on $V$. By maximality of $I, z$ is not injective on $I \cup V$. Hence $\left.z\right|_{I \cup V}$ contains a loop, which contradicts (10.3). This proves that $z(a) \in \operatorname{tip}\left(K_{0}\right)$.

Denote by $\gamma_{1}$ and $\gamma_{2}$ the two components of $z(I) \backslash\{x\}$. We just proved that both $\gamma_{i}$ reach out of $B(x, \rho)$ for every $\rho<d=\operatorname{dist}\left(x, \operatorname{tip}\left(K_{0}\right)\right)$. This proves that $r_{3}(x) \geqslant d$, at least if $x$ is a regular point of $K$. The case when $K \cap B(x, \rho)$ is a spider centered at $x$ for some $\rho$ follows by continuity (see (10.13)). The last case when $x \in K^{\sharp}$ is impossible, by (10.10) and because (10.16) says that $r_{1}(x)>0$. So $r_{3}(x) \geqslant d$ in all cases.

Let us summarize the situation. The first inequality in (10.15) is immediate, because these is no point of $K^{\sharp}$ in a good curve or spider disk (by (10.10)). The second one is trivial because tip $\left(K_{0}\right) \subset K^{\sharp}$ (by (10.11)). We just proved the third one, and the last one follows from (10.16). So we just need to check that $r_{3}(x) \leqslant C r_{2}(x)$. It is even enough to check that $r_{3}(x) \leqslant C r_{1}(x)$, because $r_{1}(x) \leqslant r_{2}(x)$ trivially.

As before, it is enough to prove this when $x$ is a regular point of $K$, because the other case (when $x$ is a spider point) will follow from (10.13) by continuity. Choose $\rho>0$ such that $K \cap B(x, \rho)$ is a nice $C^{1}$ curve (as for the definition (10.12)), and call $\Omega_{+}, \Omega_{-}$the two components of $B(x, \rho) \backslash K$. Let $0<r<r_{3}(x)$ be given; and call $\Omega_{ \pm}(r)$ the connected component of $B(x, r) \backslash K$ that meets $\Omega_{ \pm}$. Then $\Omega_{+}(r) \neq \Omega_{-}(r)$ by definition of $r_{3}(x)$. Also, each $\Omega_{ \pm}(r)$ meets all the circles $\partial B(x, \rho), 0<\rho<r$, because of (9.17). Then Proposition 9.14 says that $B\left(x, a_{2} r\right)$ is a good curve or spider disk. Thus $r_{1}(x) \geqslant a_{2} r$ and, since we could choose any $r<r_{3}, r_{1}(x) \geqslant a_{2} r_{3}(x)$.

This completes our proof of (10.15) when $r_{2}>0$. When $r_{2}(x)=0, r_{1}(x)=0$ by definition of $r_{1}(x), x \in \operatorname{tip}\left(K_{0}\right)=K_{0} \cap K^{\sharp}$ by (10.10) and (10.11), and $r_{3}(x)=0$ by definition. In this case also (10.15) holds; Proposition 10.14 follows.

An easy consequence of Proposition 10.14 is that there exists an absolute constant $C>1$ such that if $x$ and $y$ are two distinct spider points of $K_{0}$ then

$$
|x-y| \geqslant C^{-1} \max \left(\operatorname{dist}\left(x, K^{\sharp}\right), \operatorname{dist}\left(y, K^{\sharp}\right)\right) .
$$

Indeed, if $B(x, r)$ is a good curve or spider disk, there is at most one spider point in $B(x, r)$, namely $x$. Thus $|x-y| \geqslant r$ and by definition of $r_{1}(x)$ and $r_{1}(y)$ and Proposition 10.14 we have

$$
|x-y| \geqslant \max \left(r_{1}(x), r_{1}(y)\right) \geqslant C^{-1} \max \left(\operatorname{dist}\left(x, K^{\sharp}\right), \operatorname{dist}\left(y, K^{\sharp}\right)\right) .
$$

At this point we know that $K_{0}$ is a chord-arc tree composed of $C^{1}$-curves that can only meet by sets of three and with $120^{\circ}$ angles. The tree may have extremities (which we called tips), and we cannot prove yet that there is only finitely many of them. Away from the tips, the situation is nice with uniform estimates (that is, $r_{1}(x) \geqslant C^{-1} \operatorname{dist}\left(x, \operatorname{tip}\left(K_{0}\right)\right.$ ), and in particular $K \backslash K_{0}$ does not get too close.

We end this description of nontrivial connected components of $K$ with an estimate of the jump of $u$ at regular points of $K$. Let $x \in K$ be a regular point of $K$ (as in (1.12)). It is well-known that $u(y)$ has limits $u^{ \pm}(x)$ when $y \in \mathbb{R}^{2} \backslash K$ tends to $x$ from either side of $K$. Thus we can define jump $(x)=\left|u^{+}(x)-u^{-}(x)\right|$ without ambiguity. 
PROPOSITION 10.17. - There is an absolute constant $C>1$ such that if $K$ is not a line or a propeller and $x$ is a regular point of $K$, then

$$
C^{-1} r_{1}(x)^{1 / 2} \leqslant \operatorname{jump}(x) \leqslant C r_{1}(x)^{1 / 2} .
$$

See the definition (10.7), and Proposition 10.14 for equivalents of $r_{1}(x)$. We start with the first inequality. The argument is quite standard (and for instance a minor variation of Lemma 3.19 in [7], which itself was not really new), so we shall only sketch it.

First we can apply Proposition 7.3 to the disk $B\left(x, \frac{1}{2} r_{1}(x)\right)$ and a value of $\varepsilon>0$ to be chosen later. Set $r=\frac{1}{10} a(\varepsilon) r_{1}(x)$; we get that

$$
\int_{B(x, 5 r) \backslash K}|\nabla u|^{2} \leqslant 5 \varepsilon r .
$$

The simplest case is when $K \cap B(x, r)$ is a nice $C^{1}$ curve. Choose $\rho \in\left(\frac{r}{2}, r\right)$ such that $\int_{\partial B(x, \rho) \backslash K}|\nabla u|^{2} \leqslant 10 \varepsilon$. Note that $K \cap \partial B(x, \rho)$ is composed of two points (by definition of a nice $C^{1}$ curve, see Section 7); call $I^{+}$and $I^{-}$the two components of $\partial B(x, \rho) \backslash K$. It is fairly easy to see that

$$
\left|u(y)-u^{ \pm}(x)\right| \leqslant C \sqrt{\varepsilon r} \text { for } y \in I^{ \pm},
$$

where $u^{ \pm}(x)$ denotes the boundary value of $u$ at $x$, with access from the same side of $K$ as $I^{ \pm}$. We do not give the details, but simply note that $K \cap \partial B(x, t)$ is a nice $C^{1}$ curve and the analogue of (10.19) holds for all $t<r$, which helps.

We can build a competitor $\left(u^{*}, K^{*}\right)$ for $(u, K)$ as follows. We set $K^{*}=K \backslash B\left(x, \frac{r}{4}\right)$, keep $u^{*}=u$ out of $B(x, \rho)$, and try to interpolate nicely on $B(x, \rho) \backslash K^{*}$. We can manage to get

$$
\int_{B(x, \rho) \backslash K^{*}}\left|\nabla u^{*}\right|^{2} \leqslant C \operatorname{jump}(x)^{2}+C \varepsilon r,
$$

because of (10.19) and (10.20) in particular.

We do not need to check (1.4) here, because $K$ is not a line or a propeller and hence $\mathbb{R}^{2} \backslash K$ is connected (by Corollary 9.16). Thus $\left(u^{*}, K^{*}\right)$ is an acceptable competitor and (1.6) says that

$$
\frac{r}{2} \leqslant H^{1}\left(K \cap B\left(x, \frac{r}{4}\right)\right) \leqslant \int_{B(x, \rho) \backslash K^{*}}\left|\nabla u^{*}\right|^{2} \leqslant C \operatorname{jump}(x)^{2}+C \varepsilon r,
$$

which implies the first inequality in (10.18) if $\varepsilon$ is small enough.

We are left with the case when $K \cap B(x, r)$ is not a nice $C^{1}$ curve. However $B(x, r)$ is a good curve or spider disk; this follows from the heredity property of good curve or spider disks mentionned just after the definition in Section 7. Then $K \cap B(x, 4 r)$ is a nice spider, with its center in $B(x, 2 r)$. We can proceed essentially as before, except that in the definition of $K^{*}$ we only remove the part of $K \cap B(x, r / 2)$ which lies on the same leg of the spider as $x$. We omit the details. 
Now we turn to the second half of (10.18). Because of Proposition 10.14, it is enough to prove that $\operatorname{jump}(x) \leqslant C r_{3}(x)$, where $r_{3}(x)$ is defined by (10.12). There are probably direct ways to do this, but we shall apply simply Lemma 21.3 in [5].

In this lemma we are given a disk $B=B\left(x_{0}, r_{0}\right)$ and a $C^{1}$ function $f$ on $\mathbb{R}^{2} \backslash K$ such that

$$
|\nabla f(y)| \leqslant C_{0} r_{0}^{-1 / 2}+C_{0} \operatorname{dist}(y, K)^{-1 / 2} \text { for } y \in \mathbb{R}^{2} \backslash K .
$$

Here we shall take $f=u, x_{0}=x$, and $r_{0}=2 r_{3}(x)$. Note that $r_{3}(x)<+\infty$, because $\mathbb{R}^{2} \backslash K$ is connected. The estimate (10.23) would be easily checked, because of (2.4) and the harmonicity of $u$, but we do not even need to do this because it is done in (25.22) of [5] (and the term $C_{0} r_{0}^{-1 / 2}$ in (10.23) is not even needed).

In that same lemma that we want to apply, there are two points $y_{1}, y_{2} \in B \backslash K$, which are assumed to lie in the same connected component of $B \backslash K$. (See (21.2) in [5].) Then the conclusion of the lemma is that

$$
\left|f\left(y_{1}\right)-f\left(y_{2}\right)\right| \leqslant C_{3} C_{0} r_{0}^{1 / 2},
$$

where $C_{3}$ is an absolute constant.

Here we apply this with points $y_{1}, y_{2}$ that tend to $x$ from both sides of $K$. They lie in the same component of $B \backslash K$ by definition of $r_{3}(x)$ and choice of $r_{0}$. Then (10.24) holds and the upper bound in (10.18) follows.

Proposition 10.17 also gives estimates on the three jumps of $u$ at a spider point, just by taking limits. Its proof also gives a reasonable control on the oscillation of $u$ in a small ball near a tip of $K_{0}$. We omit the details.

\section{Stability of the singular singular set $K^{\sharp}$}

Recall from (10.9) and (10.10) that $K^{\sharp}$ denotes the set of points of high energy, which is also the set of points $x \in K$ for which $r_{1}(x)=0$. In particular, $K^{\sharp}$ is closed.

THEOREM 11.1. - Let $\left\{\left(u_{n}, K_{n}\right)\right\}$ be a sequence of reduced global minimizers in the plane that converges to some $(u, K)$. Then the sets $K_{n}^{\sharp}$ converge to $K^{\sharp}$.

See the beginning of Section 8 for our definition of convergence. Also note that $(u, K) \in R G M$ by Theorem 8.5 ; thus it is legitimate to talk about $K^{\sharp}$.

Half of the theorem was known from ([15], Proposition 40). That is, the fact that if $\left\{K_{n}^{\sharp}\right\}$ converges to some limit $H$, then $H \subset K^{\sharp}$. For the other half we shall use Proposition 10.14 (or equivalently Theorem 9.1).

Let us prove that

$$
\lim _{n \rightarrow+\infty} \operatorname{dist}\left(x, K_{n}^{\sharp}\right)=0 \quad \text { for } x \in K^{\sharp} .
$$

Suppose not. Then we can find $x \in K^{\sharp}, \rho>0$, and an increasing sequence $\left\{n_{k}\right\}$ such that $\operatorname{dist}\left(x, K_{n_{k}}^{\sharp}\right) \geqslant \rho$ for all $k$. Without loss of generality, we can assume that $n_{k}=k$ for all $k$. This amounts to replacing $\left\{\left(u_{n}, K_{n}\right)\right\}$ with a subsequence and simplifies the notation. 
Since $K$ is the limit of $\left\{K_{n}\right\}$, we can find points $x_{n} \in K_{n}$ such that $\left\{x_{n}\right\}$ converges to $x$. Then

$$
\operatorname{dist}\left(x_{n}, K_{n}^{\sharp}\right) \geqslant \rho / 2
$$

for $n$ large enough, and Proposition 10.14 says that

$$
\begin{array}{ll}
r_{1}\left(x_{n}\right) \geqslant C^{-1} \rho & \text { for } n \text { large enough, } \\
& \text { where here } r_{1}\left(x_{n}\right) \text { is defined in terms of } K_{n} .
\end{array}
$$

Let $\varepsilon_{0}$ be as in Theorem 7.1, then set $\varepsilon=\varepsilon_{0} / 2$ and apply Proposition 7.3 to $\left(u_{n}, K_{n}\right)$ and the disk $B\left(x_{n}, \frac{\rho}{2 C}\right)$, where $C$ is as in (11.4). We get that

$$
\int_{B\left(x_{n}, t\right) \backslash K_{n}}\left|\nabla u_{n}\right|^{2} \leqslant \varepsilon t,
$$

with $t=a(\varepsilon) \frac{\rho}{2 C}$. Since $B(x, t / 2) \subset B\left(x_{n}, t\right)$ for $n$ large and $\left\{\nabla u_{n}\right\}$ converges to $\nabla u$ uniformly on compact subsets of $\mathbb{R} \backslash K$, Fatou's lemma says that

$$
\int_{B(x, t / 2) \backslash K}|\nabla u|^{2} \leqslant \varepsilon t .
$$

Then Theorem 7.1 says that $B\left(x, \frac{a_{0} t}{2}\right)$ is a good curve or spider disk, and obviously $x \notin K^{\sharp}$. This contradiction proves (11.2).

Now let $x \in K \backslash K^{\sharp}$ be given, set $d=\operatorname{dist}\left(x, K^{\sharp}\right)$, and let us check that

$$
\operatorname{dist}\left(x, K_{n}^{\sharp}\right) \geqslant \frac{d}{C_{1}} \quad \text { for } n \text { large, }
$$

where $C_{1}$ is an absolute constant. This is the part of the argument that is very close to Proposition 40 in [15], but we sketch it anyway.

First note that $r_{1}(x) \geqslant d / C$, by Proposition 10.14. Then apply Proposition 7.3 with $\varepsilon=\varepsilon_{0} / 3$ to the disk $B(x, d / C)$. We get that

$$
\int_{B(x, t) \backslash K}|\nabla u|^{2} \leqslant \frac{\varepsilon_{0} t}{3},
$$

where we set $t=\frac{a(\varepsilon) d}{2 C}$. Then we use Theorem 2.2 in [4] (as we did for (9.11)) to get that

$$
\int_{B(x, t) \backslash K_{n}}\left|\nabla u_{n}\right|^{2} \leqslant \frac{\varepsilon_{0} t}{2}
$$

for $n$ large. As before, choose $x_{n} \in K_{n}$ so that $\left\{x_{n}\right\}$ converges to $x$. Then

$$
\int_{B\left(x_{n}, t / 2\right) \backslash K_{n}}\left|\nabla u_{n}\right|^{2} \leqslant \frac{\varepsilon_{0} t}{2}
$$


for $n$ large, just by (11.9). Theorem 7.1 says that $B\left(x_{n}, \frac{a_{0} t}{2}\right)$ is a good curve or spider disk for $K_{n}$, and in particular $\operatorname{dist}\left(x_{n}, K_{n}^{\sharp}\right) \geqslant \frac{a_{0} t}{2}=\frac{a_{0} a(\varepsilon) d}{4 C}$. This proves (11.7).

The convergence of $\left\{K_{n}^{\sharp}\right\}$ to $K^{\sharp}$ easily follows from (11.2), (11.7), and the fact that $\left\{K_{n}\right\}$ converges to $K$. The main point is that if $z_{n} \in K_{n}^{\sharp}$ for $n \geqslant 0$ and $\left\{z_{n}\right\}$ is bounded, then we can extract a subsequence of $\left\{z_{n}\right\}$ that converges to some $x \in K$, and (11.7) forces $x$ to lie on $K^{\sharp}$. The theorem follows.

Let us say that the minimizer $(u, K) \in R G M$ is exotic when it is not one of the usual minimizers that are described in (1.8)-(1.11). Recall from [15] Proposition 45 that $K^{\sharp}$ is unbounded when $(u, K)$ is exotic. We want to use Theorem 11.1 to improve slightly on this.

PROPOSITION 11.11. - There is an absolute constant $C>1$ such that if $(u, K) \in$ $R G M$ and $x \in K^{\sharp}$, then

$$
\begin{aligned}
& K^{\sharp} \text { meets the annulus }\left\{z \in \mathbb{R}^{2} ; \lambda<|z-x|<C \lambda\right\} \\
& \text { for every } \lambda>\operatorname{dist}\left(x, K^{\sharp} \backslash\{x\}\right) .
\end{aligned}
$$

Here we did not specify that $(u, K)$ is exotic, but if it is not, then $K^{\sharp}$ is empty or reduced to one point and $\operatorname{dist}\left(x, K^{\sharp} \backslash\{x\}\right)=+\infty$, and the proposition is empty.

We want to prove this by contradiction and compactness, so let us assume that for each integer $n>1$ we can find a minimizer $\left(u_{n}, K_{n}\right)$, a point $x_{n} \in K_{n}^{\sharp}$, and a radius $\lambda_{n}>d_{n}=\operatorname{dist}\left(x_{n}, K_{n}^{\sharp} \backslash\left\{x_{n}\right\}\right)$ for which (11.12) fails with $C=n$.

We can assume that $x_{n}=0$ (otherwise, translate everything). Since $\lambda_{n}>d_{n}$, we can also assume that $K_{n}^{\sharp} \cap \partial B\left(0, \lambda_{n}\right)$ is not empty (otherwise, replace the annulus in (11.12) by a similar one with a smaller $\lambda$ ). We may also assume that $\lambda_{n}=1$, because everything is invariant under dilations.

Now take a subsequence of $\left\{\left(u_{n}, K_{n}\right)\right\}$ that converges. This is possible, by Lemma 8.3, and the limit $\left(u_{\infty}, K_{\infty}\right)$ lies in RGM, by Theorem 8.5. Moreover, Theorem 11.1 says that $K_{\infty}^{\sharp}$ is the limit of the corresponding subsequence of $\left\{K_{n}^{\sharp}\right\}$. In particular $0 \in K_{\infty}^{\sharp}$ and $K_{\infty}^{\sharp}$ meets $\partial B(0,1)$, and so $\left(u_{\infty}, K_{\infty}\right)$ is exotic. On the other hand, $K_{n}^{\sharp}$ does not meet $\left\{z \in \mathbb{R}^{2} ; 1<|z|<n\right\}$, and hence $K_{\infty}^{\sharp} \subset \bar{B}(0,1)$. This contradicts the above mentionned Proposition 45 from [15], and Proposition 11.11 follows.

Let us rapidly see, for the sake of completeness, how to prove that

$$
K^{\sharp} \text { is unbounded when }(u, K) \text { is exotic. }
$$

First note that

every connected component of $K$ contains at least one point of $K^{\sharp}$.

For components that are reduced to one point, this comes directly from (10.10). For other components $K_{0}$, notice that otherwise Proposition 10.14 would say that $r_{1}(x)=+\infty$ for all $x \in K_{0}$, and hence that $K$ is a line or a propeller (for instance because it is connected).

Now $K^{\sharp}$ cannot be empty (because of (11.14)). It cannot be reduced to one point either, because then (11.14) would say that $K$ is connected. Finally assume that $K^{\sharp}$ is 
bounded (but not empty), to get a contradiction. Let $\left(u_{\infty}, K_{\infty}\right)$ be any blow-in limit of $(u, K)$. This means in particular that $K_{\infty}$ is the limit of $\left\{t_{n} K\right\}$ for some sequence $\left\{t_{n}\right\}$ that tends to 0 . Then Theorem 11.1 says that $K_{\infty}^{\sharp}=\{0\}$. Hence $\left(u_{\infty}, K_{\infty}\right)$ is a cracktip (as in (1.11)) because, as we just said, an exotic minimizer would not have $K_{\infty}^{\sharp}=\{0\}$. Now we can apply Proposition 40.5 in [5], which says that $(u, K)$ also is a cracktip. This gives the desired contradiction.

\section{Global minimizers in a cone}

In this section we give a complete description of the reduced global minimizers in the cones

$$
C_{\alpha}=\left\{\rho \mathrm{e}^{\mathrm{i} \theta} ; \rho>0 \text { and } 0<\theta<\alpha\right\}
$$

with aperture $\alpha<3 \pi / 2$.

The definition of global minimizers in $C_{\alpha}$ is the same as for the plane. Admissible pairs are pairs $(u, K)$ for which $K$ is a (relatively) closed subset of $C_{\alpha}, u \in W_{\text {loc }}^{1,2}\left(C_{\alpha} \backslash K\right)$, and the analogue of (1.1) with $\mathbb{R}^{2}$ replaced by $C_{\alpha}$ holds. The definitions of competitors for $(u, K)$, global minimizers, and then reduced global minimizers in $C_{\alpha}$ are the same as before, except that we replace $\mathbb{R}^{2}$ with $C_{\alpha}$ everywhere. We shall denote by $\operatorname{RGM}\left(C_{\alpha}\right)$ the set of reduced global minimizers in $C_{\alpha}$.

We want to show that when $\alpha<3 \pi / 2, R G M\left(C_{\alpha}\right)$ reduces to the following trivial examples. First we can take $K=\emptyset$ and $u$ constant on $C_{\alpha}$. We can also let $K$ be a halfline with its origin in $\partial C_{\alpha}$ and $u$ be constant on each component of $C_{\alpha} \backslash K$. It is easy to see that this gives a global minimizer in $C_{\alpha}$ if and only if the following conditions are satisfied. If the origin of $K$ lies on $\partial C_{\alpha} \backslash\{0\}, K$ must be perpendicular to $\partial C_{\alpha}$ at that point. If the origin of $K$ is $0, K$ must make angles $\geqslant \pi / 2$ with the two branches of $\partial C_{\alpha}$. Finally, $\alpha \geqslant \pi$ is needed in all cases.

THEOREM 12.2. - If $0<\alpha<3 \pi / 2$ and $(u, K) \in R G M\left(C_{\alpha}\right)$, then $K$ is empty or a half-line with its origin in $\partial C_{\alpha}$ (and the constraints explained above), and $u$ is locally constant on $C_{\alpha} \backslash K$.

A few comments on this statement may be useful. The situation in $C_{\alpha}$ is simpler because there is less room than in the plane; this will be clear in the proof. There is no reason to believe that something special happens for $\alpha=3 \pi / 2$. We claim that somewhat painful adaptations of the proof below would give values of $\alpha$ slightly larger than $3 \pi / 2$, and probably Theorem 12.2 stays true for all $\alpha<2 \pi$.

The most interesting case of Theorem 12.2 is probably when $\alpha=\pi$ and $C_{\alpha}$ is a half-plane. Then the situation looks a little simpler because global minimizers in $C_{\alpha}$ correspond by reflection to global minimizers in the plane that are symmetric with respect to the first axis. The simplification is not enormous, though.

To prove the theorem we introduce the same function $F(r)=\frac{2 E(r)+\ell(r)}{r}$ as in the planar case, where

$$
\ell(r)=H^{1}(K \cap B(0, r)) \text { and } \quad E(r)=\int_{C_{\alpha} \cap B(0, r) \backslash K}|\nabla u|^{2} .
$$


The proof of Proposition 2.5 still works here; there is an open set of full measure $\mathcal{R} \subset(0,+\infty)$ such that $F^{\prime}(r)$ exists (and can be computed as in (2.21)) for $r \in \mathcal{R}$, and

$$
F\left(r_{2}\right)-F\left(r_{1}\right) \geqslant \int_{\mathcal{R} \cap\left(r_{1}, r_{2}\right)} F^{\prime}(r) d r
$$

for $0<r_{1}<r_{2}$. Next we claim that the proof of Proposition 3.5 still applies here. The main reason for this is that the homeomorphisms $\varphi_{t}$ that were used there are radial, hence preserve $C_{\alpha}$. All this is good for us, because it allows us to go from the analogue of (2.21) to the analogue of (3.8), namely

$$
\frac{r}{2} F^{\prime}(r) \geqslant \frac{3}{2} J_{\tau}+\frac{1}{2} J_{v}+N(r)-\frac{E(r)+\ell(r)}{r},
$$

where $J_{\tau}$ and $J_{v}$ are defined as in (3.3) and (3.4), and $N(r)$ still denotes the number of points in $K \cap \partial B(0, r)$.

Proposition 2.13 also goes through (see the beginning of Section 5). Note that if $\pi \omega r$ denotes the length of the longest component of $\partial B(0, r) \cap C_{\alpha} \backslash K$, then $\omega \leqslant \alpha / \pi$. Thus (2.14) yields

$$
r F^{\prime}(r) \geqslant m \int_{\partial B(0, r) \cap C_{\alpha} \backslash K}|\nabla u|^{2}=m\left(J_{\tau}+J_{v}\right)
$$

for $r \in \mathcal{R}$, where we set

$$
m=\min \left(1,3-\frac{2 \alpha}{\pi}\right)>0 .
$$

This will be our main estimate, but there are a few cases where we wish to improve it. Let $r \in \mathcal{R}$ be given and call $r \mathrm{e}^{\mathrm{i} \theta_{1}}, \ldots, r \mathrm{e}^{\mathrm{i} \theta_{N}}$ the points of $K \cap \partial B(0, r)$, with $0<\theta_{1}<$ $\theta_{2}<\cdots<\theta_{N}<\alpha$. Let us check that

$$
r F^{\prime}(r) \geqslant 2\left(1-\sin \theta_{1}\right) \quad \text { when } \theta_{1}<\frac{\pi}{2} .
$$

We proceed as in Section 5 and construct a competitor $(v, L)$ for $(u, K)$, where $L \cap B(0, r)$ is composed of the $N-1$ segments $\left[0, r \mathrm{e}^{\mathrm{i} \theta_{1}}\right], i>1$, plus the shortest line segment from $r \mathrm{e}^{\mathrm{i} \theta_{1}}$ to $\partial C_{\alpha}$. See Fig. 10. The function $v$ on $B(0, r) \cap C_{\alpha} \backslash L$ can be constructed using Corollary 4.15, and we can even take $\alpha^{\prime} \leqslant 3 / 2$ there. The comparison yields

$$
\begin{aligned}
E(r)+\ell(r) & \leqslant H^{1}(L \cap B(0, r))+\int_{B(0, r) \cap C_{\alpha} \backslash L}|\nabla v|^{2} \\
& \leqslant(N-1) r+r \sin \theta_{1}+\frac{3}{2} r J_{\tau} .
\end{aligned}
$$

We plug this back into (12.5) and get (12.8). For the same reasons,

$$
r F^{\prime}(r) \geqslant 2\left(1-\sin \left(\alpha-\theta_{N}\right)\right) \quad \text { when } \theta_{N}>\alpha-\frac{\pi}{2} .
$$




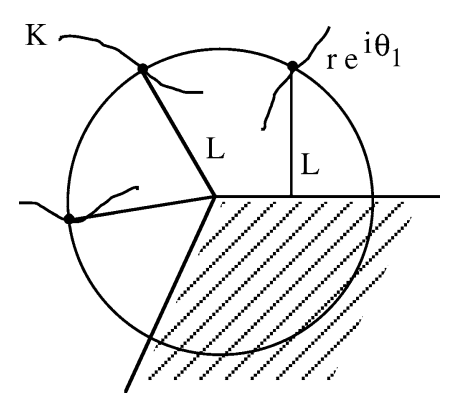

Fig. 10 .

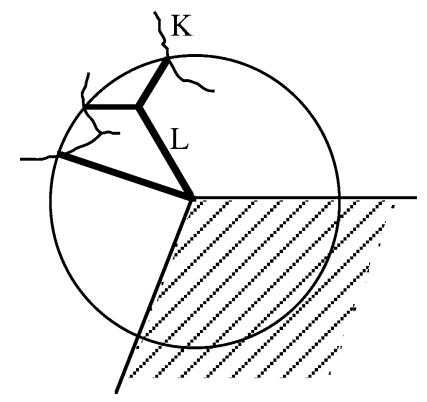

Fig. 11 .

Next we claim that

$$
r F^{\prime}(r) \geqslant 10^{-3} \text { when } N(r) \geqslant 2 .
$$

If $\theta_{1} \leqslant \frac{9 \pi}{20}$ or $\theta_{N} \geqslant \alpha-\frac{9 \pi}{20}$, this follows from (12.8) or (12.10). Otherwise $\theta_{2}-\theta_{1} \leqslant$ $\theta_{N}-\theta_{1} \leqslant \alpha-\frac{9 \pi}{10} \leqslant \frac{6 \pi}{10}$. Call $I$ the arc of $\partial B(0, r)$ between $r \mathrm{e}^{\mathrm{i} \theta_{1}}$ and $r \mathrm{e}^{\mathrm{i} \theta_{2}}$, and let $Y$ be the fork based on $I$ (as in Definition 5.17). Then $H^{1}(Y) \leqslant r f\left(\frac{6}{10}\right) \leqslant\left(2-10^{-3}\right) r$ (by Lemma 5.20 and a trivial estimate). This time we take $L \cap B(0, r)=Y \cup\left(\bigcup_{i>2}\left[0, r \mathrm{e}^{\mathrm{i} \theta_{i}}\right]\right)$, as suggested by Fig. 11. We can still apply Corollary 4.15 with $\alpha^{\prime} \leqslant 3 / 2$, and we get that

$$
E(r)+\ell(r) \leqslant H^{1}(L \cap B(0, r))+\int_{B(0, r) \cap C_{\alpha} \backslash L}|\nabla v|^{2} \leqslant N r-10^{-3} r+\frac{3}{2} r J_{\tau}
$$

(12.11) follows from this and (12.5).

We know from (12.4) and (12.6) that $F$ is nondecreasing. It is also bounded, because

$$
E(r)+\ell(r) \leqslant \alpha r \leqslant \frac{3 \pi r}{2} \text { for } r>0
$$

by the usual cut-off argument (add $\partial B(0, r) \cap C_{\alpha}$ to $K$ and replace everything inside by a constant). Hence we can set

$$
L=\lim _{r \rightarrow+\infty} F(r) \leqslant 3 \pi
$$

We want to use (12.6) to prove that

$$
\lim _{r \rightarrow+\infty} \frac{E(r)}{r}=0
$$

Let $\lambda>1$ be given. Note that

$$
\int_{r}^{\lambda r} F^{\prime}(t) d t \leqslant F(\lambda r)-F(r) \leqslant L-F(r)
$$


by (12.4). Then

$$
E(\lambda r)-E(r)=\int_{r}^{\lambda r}\left\{\int_{\partial B(0, t) \cap C_{\alpha} \backslash K}|\nabla u|^{2}\right\} d t \leqslant \int_{r}^{\lambda r} \frac{t}{m} F^{\prime}(t) d t \leqslant \frac{\lambda r}{m}(L-F(r))
$$

by (12.6) and (12.7). Since $L-F(r)$ tends to 0 when $r$ tends to $+\infty$, we get that $\frac{E(\lambda r)-E(r)}{\lambda r}$ also tends to 0 . Since $\frac{E(r)}{\lambda r} \leqslant \frac{3 \pi}{2 \lambda}$ by (12.13), we get that $\lim _{\sup } \sin _{\rho \rightarrow+\infty} \frac{E(\rho)}{\rho} \leqslant \frac{3 \pi}{2 \lambda}$ for any given $\lambda>1 ;$ (12.15) follows.

Let $\varepsilon>0$ be given, and let $r \in \mathcal{R}$ be such that $r F^{\prime}(r) \leqslant \varepsilon$. Because of (12.16) (with $\lambda=2$ ), Tchebychev, and (12.14) we know that we can find arbitrarily large radii $r$ like this. Note that $N(r) \leqslant 1$ (if $\varepsilon<10^{-3}$ ), by (12.11).

If $N(r)=1$, we compare $(u, K)$ with the usual competitor $(v, L)$ for which $L=$ $\left[0, r \mathrm{e}^{\mathrm{i} \theta_{1}}\right] \cup(K \backslash B(0, r))$ and $v$ is given by Corollary 4.15. We get that

$$
E(r)+\ell(r) \leqslant r+\int_{B(0, r) \backslash L}|\nabla v|^{2} \leqslant r+\frac{3}{2} r J_{\tau} \leqslant r+\frac{3 \varepsilon r}{2 m},
$$

by (12.6) and because $r F^{\prime}(r) \leqslant \varepsilon$.

If $N(r)=0$ we can use $L=K \backslash B(0, r)$ and Lemma 4.10, which yields

$$
E(r)+\ell(r) \leqslant \frac{3}{2} r J_{\tau} \leqslant \frac{3 \varepsilon r}{2 m} .
$$

Note that $L=\lim _{r \rightarrow+\infty} \frac{\ell(r)}{r}$, by (12.14) and (12.15). Since for each small $\varepsilon>0$ we can find arbitrarily large radii $r$ for which (12.18) or (12.19) holds (and hence $\frac{\ell(r)}{r} \leqslant 1+\frac{3 \varepsilon}{2 m}$ ), we see that $L \leqslant 1$. Let us check that

$$
K=\emptyset \text { and } u \text { is constant if } L<1 .
$$

Set $\delta=\frac{1-L}{3}>0$ and $\lambda=\delta^{-1}$. Since $L=\lim _{r \rightarrow+\infty} \frac{\ell(r)}{r}$ we know that for $r$ large enough

$$
H^{1}(K \cap B(0, \lambda r))=\ell(\lambda r) \leqslant(1-2 \delta) \lambda r=\lambda r-2 r .
$$

Set $E_{0}=\{t \in(0, \lambda r) ; N(t)=0\}$ and $E_{1}=(0, \lambda r) \backslash E_{0}$. Then $E_{1}$ is the image of $K \cap B(0, \lambda r)$ under the 1-Lipschitz mapping $z \rightarrow|z|$. Consequently $H^{1}\left(E_{1}\right) \leqslant \lambda r-2 r$, by (12.21), and $H^{1}\left(E_{0}\right) \geqslant 2 r$. Since $\mathcal{R}$ has full measure, $H^{1}\left(\mathcal{R} \cap E_{0} \cap(r, \lambda r)\right) \geqslant r$. By (12.16) and Tchebychev, we can find $t \in \mathcal{R} \cap E_{0} \cap(r, \lambda r)$ such that $F^{\prime}(t) \leqslant$ $(L-F(r)) / r$.

Let $\varepsilon>0$ be small. If $r$ is large enough, $L-F(r) \leqslant \varepsilon / \lambda$ and $t F^{\prime}(t) \leqslant \frac{t \varepsilon}{\lambda r} \leqslant \varepsilon$. Then (12.19) holds for $t$ (because $N(t)=0)$ and $E(t)+\ell(t) \leqslant \frac{3 \varepsilon t}{2 m}$. Thus we found arbitrarily large radii $t$ for which $F(t) \leqslant \frac{6 \varepsilon}{2 m}$. This proves that $L=0$, but then $F(t) \equiv 0$ because $F$ is nondecreasing; (12.20) follows.

We may now restrict our attention to the remaining case when

$$
L=\lim _{r \rightarrow+\infty} \frac{\ell(r)}{r}=1 .
$$




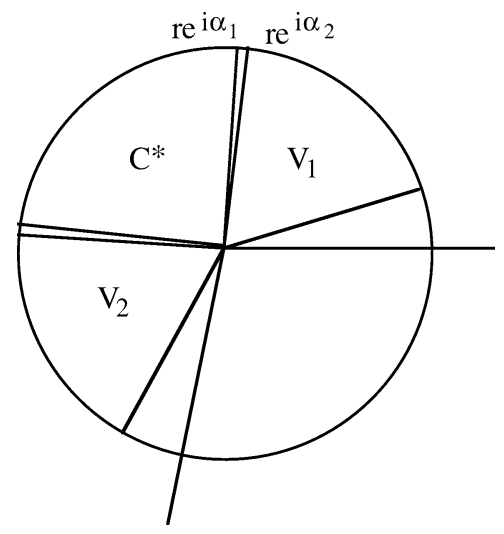

Fig. 12.

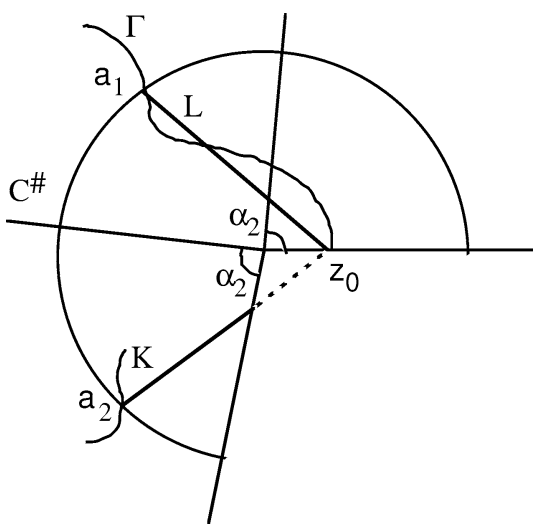

Fig. 13.

Next we wish to show that most of $K$ lies in the smaller cone

$$
C^{*}=\left\{\rho \mathrm{e}^{\mathrm{i} \theta} ; \rho>0 \text { and } \alpha_{1}<\theta<\alpha-\alpha_{1}\right\}
$$

where $\alpha_{1} \in\left(\frac{9 \pi}{20}, \frac{\pi}{2}\right)$ will be chosen later. See Fig. 12 already. The reader should not worry: the argument below will show that $\alpha>2 \alpha_{1}$ and $C^{*}$ is not empty. First set

$$
\mathcal{R}(\varepsilon)=\left\{t \in \mathcal{R} ; t F^{\prime}(t) \leqslant \varepsilon\right\}
$$

for $\varepsilon>0$, and let us check that

$$
\lim _{r \rightarrow+\infty} \frac{1}{r} H^{1}([r, 2 r] \backslash \mathcal{R}(\varepsilon))=0
$$

for each $\varepsilon>0$. Set $Z(r)=[r, 2 r] \backslash \mathcal{R}(\varepsilon)$; then (12.16) says that

$$
\frac{1}{r} H^{1}(Z(r)) \leqslant \int_{Z(r)} \frac{t F^{\prime}(t)}{r \varepsilon} d t \leqslant \frac{2}{\varepsilon} \int_{Z(r)} F^{\prime}(t) d t \leqslant \frac{2}{\varepsilon}(L-F(r)),
$$

which tends to 0 when $r$ tends to $+\infty$.

LEMMA 12.27. - If $\varepsilon$ is small enough,

$$
\begin{aligned}
& N(t)=1 \text { and the point of } K \cap \partial B(0, t) \text { lies in } C^{*} \\
& \text { when } t \in \mathcal{R}(\varepsilon) \text { is large enough. }
\end{aligned}
$$

Indeed (12.11) says that $N(t) \leqslant 1$. If $t$ is large enough, $\ell(t) \geqslant t / 2$ (because of (12.22)), and then (12.19) cannot hold for $t$. Hence $N(t)=1$. Finally the point of $K \cap \partial B(0, t)$ lies in $C^{*}$ (if $\varepsilon$ is small enough) because $\alpha_{1}<\pi / 2$ and by (12.8) and (12.10). 
LEMMA 12.29. - For each $\alpha_{2} \in\left(\frac{9 \pi}{20}, \alpha_{1}\right)$ we can find $R>0$ such that $K \cap\left(V_{1} \cup V_{2}\right) \subset$ $B(0, R)$, where

$$
\begin{gathered}
V_{1}=\left\{\rho \mathrm{e}^{\mathrm{i} \theta} ; \rho>0 \text { and } \frac{\pi}{10}<\theta<\alpha_{2}\right\}, \\
V_{2}=\left\{\rho \mathrm{e}^{\mathrm{i} \theta} ; \rho>0 \text { and } \alpha-\alpha_{2}<\theta<\alpha-\frac{\pi}{10}\right\} .
\end{gathered}
$$

Set $A(r)=B(0,2 r) \backslash B(0, r)$ for $r>0$, and call $\pi(z)=|z|$ the radial projection of any $z \in \mathbb{R}^{2}$. Lemma 12.27 says that for $r$ large, $\pi\left(K \cap A(r) \cap C^{*}\right)$ contains $[r, 2 r) \cap \mathcal{R}(\varepsilon)$. Then

$$
\frac{1}{r} H^{1}\left[K \cap A(r) \cap C^{*}\right] \geqslant \frac{1}{r} H^{1}\left(\pi\left[K \cap A(r) \cap C^{*}\right]\right) \geqslant \frac{1}{r} H^{1}([r, 2 r) \cap \mathcal{R}(\varepsilon)),
$$

which tends to 1 by (12.25). Also,

$$
\lim _{r \rightarrow+\infty} \frac{1}{r} H^{1}(K \cap A(r))=\lim _{r \rightarrow+\infty} \frac{\ell(2 r)-\ell(r)}{r}=1,
$$

by (12.22). Consequently,

$$
\lim _{r \rightarrow+\infty} \frac{1}{r} H^{1}\left(K \cap A(r) \backslash C^{*}\right)=0 .
$$

Now let $z$ be any point of $K \cap\left(V_{1} \cup V_{2}\right)$, and set $r=\frac{2}{3}|z|, \quad r_{1}=\frac{1}{2}\left(\alpha_{1}-\alpha_{2}\right) r$, and $B=B\left(z, r_{1}\right)$. Then $B \subset C_{\alpha}$, and the local Ahlfors-regularity of $K$ says that $H^{1}(K \cap B) \geqslant C^{-1} r$. (We do not care if $C$ depends on $\alpha, \alpha_{1}$, and $\alpha_{2}$.) On the other hand, $B \subset A(r) \backslash C^{*}$, hence (12.34) forbids $r$ to be too large. The lemma follows.

LEMmA 12.35. - The sets $V_{1} \backslash B(0, R)$ and $V_{2} \backslash B(0, R)$ lie in two different components of $C_{\alpha} \backslash K$.

The idea is that otherwise we can construct a better competitor for $(u, K)$ by removing $K \cap B(0, t)$ for large values of $t$. First we want to show that $u$ does not grow too fast in $V_{1}$ and $V_{2}$.

Set $\theta_{1}=\frac{\pi}{4}, \theta_{2}=\alpha-\frac{\pi}{4}$, and then $z_{j}(t)=t \mathrm{e}^{\mathrm{i} \theta j}$ and $f_{j}(t)=u\left(z_{j}(t)\right)$ for $j=1,2$ and $t$ large. Note that $\operatorname{dist}\left(z_{j}(t), K \cup \partial C_{\alpha}\right) \geqslant \frac{t}{2}$ for $t$ large, by Lemma 12.29. Since $u$ is harmonic, we get that

$$
\left|f_{j}^{\prime}(t)\right| \leqslant \frac{C}{t}\left\{\int_{B\left(z_{j}(t), \frac{t}{2}\right)}|\nabla u|^{2}\right\}^{1 / 2} \leqslant \frac{C E(2 t)^{1 / 2}}{t}
$$

for $t$ large enough. Then (12.15) says that $\lim _{t \rightarrow+\infty}\left(t^{1 / 2} f_{j}^{\prime}(t)\right)=0$, and hence

$$
\lim _{t \rightarrow+\infty} t^{-1 / 2} f_{j}(t)=0 .
$$

Let $\varepsilon>0$ be small, to be chosen later, and let $r$ be large. First choose $t \in(r, 2 r) \cap$ $\mathcal{R}(\varepsilon)$; this is possible, by (12.25). If $\varepsilon$ is small enough, Lemma 12.27 says that $N(t)=1$ 
and that if $z_{0}$ denotes the point of $K \cap \partial B(0, t)$, then $z_{0} \in C^{*}$. We also know that

$$
\int_{\partial B(0, t) \cap C_{\alpha} \backslash\left\{z_{0}\right\}}|\nabla u|^{2} \leqslant m^{-1} t F^{\prime}(t) \leqslant m^{-1} \varepsilon,
$$

by (12.6) and (12.24). Next (12.37) says that

$$
\left|f_{1}(t)-f_{2}(t)\right| \leqslant \varepsilon^{1 / 2} t^{1 / 2}
$$

if $r$ is large enough, so the values of $u$ on the two components of $\partial B(0, t) \cap C_{\alpha} \backslash\left\{z_{0}\right\}$ are not so different.

Set $K_{1}=(K \backslash B(0, t)) \cup\left[\frac{1}{2} z_{0}, z_{0}\right]$. Then

$$
H^{1}\left(K_{1} \cap B(0, t)\right)=\frac{t}{2} \leqslant \ell(t)-\frac{t}{3}=H^{1}(K \cap B(0, t))-\frac{t}{3}
$$

for $r$ large, by (12.22). On the other hand, (12.38) and (12.39) allow us to construct a smooth function $u_{1}$ on $\bar{B}(0, t) \cap C_{\alpha} \backslash K_{1}$ that coincides with $u$ on $\partial B(0, t) \cap C_{\alpha} \backslash\left\{z_{0}\right\}$ and for which

$$
\int_{B(0, t) \cap C_{\alpha} \backslash K_{1}}\left|\nabla u_{1}\right|^{2} \leqslant C \varepsilon t .
$$

Now $\left(u_{1}, K_{1}\right)$ cannot be a competitor for $(u, K)$, because otherwise (12.40) and (12.41) would contradict the analogue of (1.6) (if $\varepsilon$ is small enough). Hence our topological condition (1.4) is violated, and the only way this can happen is that the two components of $\partial B(0, t) \cap C_{\alpha} \backslash\left\{z_{0}\right\}$ lie in different components of $C_{\alpha} \backslash K$.

Lemma 12.35 follows, because we already know that $\left(V_{1} \cup V_{2}\right) \backslash B(0, R) \subset C_{\alpha} \backslash K$.

Next we want to find a curve in $K$ that goes from $\partial C_{\alpha}$ to infinity. Choose $t_{1}>R$ such that $t_{1} \in \mathcal{R}(\varepsilon)$, where $\varepsilon$ is sufficiently small for Lemma 12.27 to apply. Call $I_{1}$ and $I_{2}$ the two components of $\partial B\left(0, t_{1}\right) \cap C_{\alpha} \backslash K$. Since each $V_{i} \backslash B(0, R)$ meets $I_{1}$ or $I_{2}$, Lemma 12.35 tells us that $K$ separates $I_{1}$ from $I_{2}$ in $C_{\alpha}$. Then $\left(K \cup \partial C_{\alpha}\right) \cap \bar{B}\left(0, t_{1}\right)$ separates $I_{1}$ from $I_{2}$ in $\bar{B}\left(0, t_{1}\right)$, and Theorem 14.3 on p. 123 of [20] says that we can find a connected piece $\Gamma_{0} \subset\left(K \cup \partial C_{\alpha}\right) \cap \bar{B}\left(0, t_{1}\right)$ that still separates them in $\bar{B}\left(0, t_{1}\right)$. Since $H^{1}\left(\Gamma_{0}\right)<+\infty$, there is a rectifiable curve $\Gamma_{1} \subset K \cap B\left(0, t_{1}\right)$ (except for its two endpoints) and which connects some point of $\partial C_{\alpha}$ to $z_{1}$, the point of $K \cap \partial B\left(0, t_{1}\right)$.

Choose $t_{2}>t_{1}+1$ such that $t_{2} \in \mathcal{R}(\varepsilon)$. There is a curve $\Gamma_{2}$ in $K \cap B\left(0, t_{2}\right) \backslash B\left(0, t_{1}\right)$ that connects the point of $K \cap B\left(0, t_{2}\right)$ to $z_{1}$. The argument is the same as for $\Gamma_{1}$. We could also construct a curve like $\Gamma_{1}$, notice that the curve has to go through $z_{1}$ (because Lemma 12.29 prevents it from getting close to $\partial C_{\alpha}$ before), and remove the part in $B\left(0, t_{1}\right)$.

We can iterate this procedure and get a curve $\Gamma \subset K$ that starts from $\partial C_{\alpha}$ and goes to infinity. Note that

$$
\Gamma \backslash B\left(0, t_{1}\right) \subset C^{\sharp}=:\left\{\rho \mathrm{e}^{\mathrm{i} \theta} ; \rho>0 \text { and } \alpha_{2} \leqslant \theta \leqslant \alpha-\alpha_{2}\right\},
$$

by Lemma 12.29 and because $z_{1} \in C^{\sharp}$. 
Call $z_{0} \in \partial C_{\alpha}$ the initial point of $\Gamma$. We want to do a last monotonicity argument, but this time with disks centered at $z_{0}$. Set $\ell_{1}(r)=H^{1}\left(K \cap B\left(z_{0}, r\right)\right), \quad E_{1}(r)=$ $\int_{B\left(z_{0}, r\right) \cap C_{\alpha} \backslash K}|\nabla u|^{2}$, and $F_{1}(r)=\left[E_{1}(r)+\ell_{1}(r)\right] / r$ for $r>0$.

We want to show that

$$
F_{1} \text { is nondecreasing on }\left(R_{1},+\infty\right)
$$

for some $R_{1}>0$. By the same sort of arguments as in Section 2,

$$
E_{1}^{\prime}(r)=\int_{\partial B\left(z_{0}, r\right) \cap C_{\alpha} \backslash K}|\nabla u|^{2}
$$

and

$$
\ell_{1}^{\prime}(r) \geqslant N_{1}(r)
$$

almost-everywhere and in the sense of distributions, where $N_{1}(r)$ denotes the number of points of $K \cap \partial B\left(z_{0}, r\right)$. Thus it will be enough to show that

$$
E_{1}(r)+\ell_{1}(r) \leqslant r N_{1}(r)+r \int_{\partial B\left(z_{0}, r\right) \cap C_{\alpha} \backslash K}|\nabla u|^{2}
$$

almost-everywhere on $\left(R_{1},+\infty\right)$.

To prove (12.46) we want to compare $(u, K)$ with a competitor $(v, L)$. As usual, we keep $(v, L)=(u, K)$ out of $B\left(z_{0}, r\right)$. Call $a_{1}, \ldots, a_{N}$ the points of $K \cap \partial B\left(z_{0}, r\right)$, and set

$$
L \cap \bar{B}\left(z_{0}, r\right)=C_{\alpha} \cap\left(\bigcup_{i=1}^{N}\left[z_{0}, a_{i}\right]\right),
$$

as suggested by Fig. 13 .

If $r$ is large enough, $K \cap \partial B\left(z_{0}, r\right)$ contains at least a point of $\Gamma$, which lies in $C^{\sharp}$ by (12.42). Choose $\alpha_{1}$ and $\alpha_{2}$ so close to $\pi / 2$ that $\alpha-\alpha_{2}<\pi$. Then for $r$ large enough, all the components of $\partial B\left(z_{0}, r\right) \cap C_{\alpha} \backslash K$ have lengths less than $\pi r$ (because of our point of $\Gamma$ ).

This allows us to use Corollary 4.15, with domains contained in half disks, to construct a function $v$ on $\bar{B}\left(z_{0}, r\right) \cap C_{\alpha} \backslash L$ that coincides with $u$ on $\partial B\left(z_{0}, r\right) \cap C_{\alpha} \backslash K$ and for which

$$
\int_{B\left(z_{0}, r\right) \cap C_{\alpha} \backslash L}|\nabla v|^{2} \leqslant r \int_{\partial B\left(z_{0}, r\right) \cap C_{\alpha} \backslash K}|\nabla u|^{2} .
$$

The comparison with $(u, K)$ yields (12.46), and then (12.43).

Set $L_{1}=\lim _{r \rightarrow+\infty} F_{1}(r)$. Then $L_{1}=1$, by (12.15) and (12.22). Hence $F_{1}(r) \leqslant 1$ for $r>R_{1}$, by (12.43). On the other hand, we have a curve $\Gamma \subset K$ that starts from $z_{0}$ and goes to infinity, so

$$
r \leqslant H^{1}\left(\Gamma \cap B\left(z_{0}, r\right)\right) \leqslant \ell_{1}(r) \leqslant r F_{1}(r)
$$


for all $r>0$. Hence all these numbers are equal, $K=\Gamma, \Gamma$ is a half line, and $u$ is locally constant (because $E_{1}(r)=0$ for $r$ large). Our proof of Theorem 12.2 is complete.

\section{Boundary behaviour of Mumford-Shah minimizers in a smooth domain}

Let $\Omega \subset \mathbb{R}^{2}$ be a bounded $C^{1}$ domain, ant let $g \in L^{\infty}(\Omega)$ be given. The MumfordShah functional is defined by

$$
J(u, K)=\int_{\Omega \backslash K}|\nabla u|^{2}+\int_{\Omega \backslash K}|u-g|^{2}+H^{1}(K),
$$

where the competitors are pairs $(u, K)$ such that $K$ is closed in $\Omega$ and $u \in W^{1,2}(\Omega \backslash K)$. Minimizers for $J$ are known to exist [1,11]. The Mumford-Shah conjecture that says that for reduced minimizers $K$ is a finite union of $C^{1}$ curve is still open, but surprisingly the situation at the boundary of $\Omega$ is much simpler. We want to rapidly explain why.

Let $(u, K)$ be a reduced minimizer for $J$, and let $x_{0} \in \partial \Omega$ be given. We want to study blow-up limits of $(u, K)$ at $x_{0}$, so we define pairs $\left(u_{r}, K_{r}\right)$ by

$$
K_{r}=r^{-1}\left(K-x_{0}\right), \quad \Omega_{r}=r^{-1}\left(\Omega-x_{0}\right),
$$

and

$$
u_{r}(x)=r^{-1 / 2} u\left(r\left(x-x_{0}\right)\right) \quad \text { for } x \in \Omega_{r} \backslash K_{r}
$$

for $r>0$ small.

Each $\left(u_{r}, K_{r}\right)$ is a reduced minimizer for a functional like $J$ on $\Omega_{r}$, but for simple reasons of homogeneity the error term $\int_{\Omega_{r} \backslash K_{r}}\left|u_{r}-g_{r}\right|^{2}$ is multiplied by a constant that tends to 0 with $r$.

Just like in Section 8, one can show that for each sequence $\left\{r_{k}\right\}$ that tends to 0 , we can extract a subsequence of $\left\{\left(u_{r_{k}}, K_{r_{k}}\right)\right\}$ that converges to some limit $\left(u_{\infty}, K_{\infty}\right)$, and that when this happens $\left(u_{\infty}, K_{\infty}\right)$ is a reduced global minimizer in some half-plane $P$. (Here we use the fact that $\partial \Omega$ has a tangent at $x_{0}$.) We claim that the proof is similar to the argument in [4], but unfortunalely we do not know of a good reference yet, and do not wish to include a proof here.

Then we can apply Theorem 12.2 so $K_{\infty}$ is either empty or a half-line perpendicular to the boundary $\partial B$, and $u_{\infty}$ is locally constant on $P \backslash K_{\infty}$.

When $K_{\infty}$ is empty, it is not too hard to show that $x_{0}$ has a small neighborhood that does not meet $K$. For instance, one may use the local Ahlfors-regularity of $K$ near $\partial \Omega$ [16] to show that otherwise there is a nontrivial amount of $K$ very near $\partial \Omega$, and then push it out using a diffeomorphism from $\Omega$ to a slightly larger domain.

When $K_{\infty}$ is a half line perpendicular to $\partial P$, it is possible to show that $x_{0}$ has a small neighborhood where $K$ is a $C^{1}$-curve that starts from $x_{0}$ and is perpendicular to $\partial \Omega$ there. The argument is similar to those needed for Section 7, but again we do not know of a good reference yet.

Because of all this, there is a small neighborhood of $\partial \Omega$ in $\Omega$ where $K$ is composed of finitely many $C^{1}$ curves that all end up on $\partial \Omega$ perpendicularly. 
Since Theorem 12.2 also allow us to control global minimizers in cones of aperture $<3 \pi / 2$ we can do similar arguments on piecewise $C^{1}$ domains $\Omega$ with interior angles less than $3 \pi / 2$. A more amusing question would be to control what happens on boundaries of Lipschitz domains $\Omega$.

\section{REFERENCES}

[1] L. Ambrosio, Existence theory for a new class of variational problems, Arch. Rational Mech. Anal. 111 (1990) 291-322.

[2] L. Ambrosio, D. Pallara, Partial regularity of free discontinuity sets I, Ann. Scuola Norm. Sup. Pisa Cl. Sci. (4) 24 (1997) 1-38.

[3] L. Ambrosio, N. Fusco, D. Pallara, Partial regularity of free discontinuity sets II, Ann. Scuola Norm. Sup. Pisa Cl. Sci. (4) 24 (1997) 39-62.

[4] A. Bonnet, On the regularity of edges in image segmentation, Ann. Inst. H. Poincaré, Analyse Non Linéaire 13 (4) (1996) 485-528.

[5] A. Bonnet, G. David, Cracktip is a global Mumford-Shah minimizer, Astérisque, Vol. 274, SMF, 2001.

[6] G. Dal Maso, J.-M. Morel, S. Solimini, A variational method in image segmentation: Existence and approximation results, Acta Math. 168 (1992) 89-151.

[7] G. David, $C^{1}$ arcs for minimizers of the Mumford-Shah functional, SIAM. J. Appl. Math. 56 (3) (1996) 783-888.

[8] G. David, S. Semmes, Analysis of and on Uniformly Rectifiable Sets, AMS Series of Mathematical Surveys and Monographs, Vol. 38, 1993.

[9] G. David, S. Semmes, On the singular sets of minimizers of the Mumford-Shah functional, J. Math. Pures Appl. 75 (1996) 299-342.

[10] E. De Giorgi, Problemi con discontinuità libera, Int. Symp. Renato Caccioppoli, Napoli, Sept. 20-22, 1989, Ricerche Mat. (suppl.) 40 (1991) 203-214.

[11] E. De Giorgi, M. Carriero, A. Leaci, Existence theorem for a minimum problem with free discontinuity set, Arch. Rational Mech. Anal. 108 (1989) 195-218.

[12] K. Falconer, The Geometry of Fractal Sets, Cambridge University Press, 1984.

[13] H. Federer, Geometric Measure Theory, Grundlehren der Mathematischen Wissenschaften, Vol. 153, Springer-Verlag, 1969.

[14] G. Hardy, J.E. Littlewood, G. Pólya, Inequalities, Second edition, Cambridge University Press, 1952.

[15] J.-C. Léger, Flatness and finiteness in the Mumford-Shah problem, J. Math. Pures Appl. (9) 78 (4) (1999) 431-459.

[16] F.A. Lops, F. Maddalena, S. Solimini, Hölder continuity conditions for the solvability of Dirichlet problems involving functionals with free discontinuities, Ann. Inst. Henri Poincaré, Anal. Non Linéaire 18 (2001) 639-673.

[17] F. Maddalena, S. Solimini, Blow-up techniques and regularity near the boundary for free discontinuity problems, Advanced Nonlinear Studies 1 (2) (2001).

[18] P. Mattila, Geometry of Sets and Measures in Euclidean Space, Cambridge Studies in Advanced Mathematics, Vol. 44, Cambridge University Press, 1995.

[19] D. Mumford, J. Shah, Optimal approximations by piecewise smooth functions and associated variational problems, Comm. Pure Appl. Math. 42 (1989) 577-685.

[20] M.H.A. Newman, Elements of the Topology of Plane Sets of Points, Second edition, reprinted, Cambridge University Press, New York, 1961. 